\title{
Pseudo-Anosov homeomorphisms and the lower central series of a surface group
}

\author{
JUSTIN MALESTEIN
}

\begin{abstract}
Let $\Gamma_{k}$ be the lower central series of a surface group $\Gamma$ of a compact surface $S$ with one boundary component. A simple question to ponder is whether a mapping class of $S$ can be determined to be pseudo-Anosov given only the data of its action on $\Gamma / \Gamma_{k}$ for some $k$. In this paper, to each mapping class $f$ which acts trivially on $\Gamma / \Gamma_{k+1}$, we associate an invariant $\Psi_{k}(f) \in \operatorname{End}\left(H_{1}(S, \mathbb{Z})\right)$ which is constructed from its action on $\Gamma / \Gamma_{k+2}$. We show that if the characteristic polynomial of $\Psi_{k}(f)$ is irreducible over $\mathbb{Z}$, then $f$ must be pseudo-Anosov. Some explicit mapping classes are then shown to be pseudo-Anosov.
\end{abstract}

57M60, 37E30

\section{Introduction}

Denote by $\operatorname{Mod}(S)$ the mapping class group of a compact, oriented surface $S=$ $S_{g, 1}$ of genus $g \geq 2$ with one boundary component; ie, $\operatorname{Mod}(S)$ is the group of homeomorphisms of $S$ fixing $\partial S$ pointwise up to isotopies fixing $\partial S$ pointwise. A basic question to contemplate is: what topological or dynamical data of a mapping class can be extracted from various kinds of algebraic data? The most complex kind of mapping class is a pseudo-Anosov mapping class, ie, a mapping class that has a representative homeomorphism which leaves invariant a pair of transverse measured foliations (see Farb and Margalit [7] for more information on pseudo-Anosov mapping classes). Thus, it is a natural question to ask if a given mapping class is pseudo-Anosov.

One kind of algebraic data is the action of a mapping class on $\Gamma:=\pi_{1}(S, *)$ and its various quotients. Specifically, consider the sequence of $k$-step nilpotent quotients $N_{k}:=\Gamma / \Gamma_{k+1}$ where $\left\{\Gamma_{k}\right\}$ is the lower central series of $\Gamma$ defined inductively by:

$$
\Gamma_{1}=\Gamma \quad \Gamma_{k}=\left[\Gamma, \Gamma_{k-1}\right] \text { for } k>1
$$

Since elements of $\operatorname{Mod}(S)$ fix $\partial S$ pointwise and we choose the basepoint $* \in \partial S$, we obtain a representation $\operatorname{Mod}(S) \rightarrow \operatorname{Aut}(\Gamma)$, and furthermore since each $\Gamma_{k}$ is characteristic, we obtain a representation for each $k$ :

$$
\rho_{k}: \operatorname{Mod}(S) \rightarrow \operatorname{Aut}\left(\Gamma / \Gamma_{k+1}\right)
$$


One natural question to ask is: given only the datum of $\rho_{k}(f)$ for $f \in \operatorname{Mod}(S)$, can we determine if the mapping class is pseudo-Anosov or not? If the mapping class is determined to be pseudo-Anosov, can we detect the dilatation? This paper is one step in answering the first question. (This paper does not address the second question. In Farb, Leininger and Margalit [6], it is shown that $\lim _{k \rightarrow \infty} \inf \{$ dilatation of $f \mid f \in$ $\left.\operatorname{ker}\left(\rho_{k}\right)\right\}=\infty$.)

For $k \geq 1$, we define the $k$ th Torelli group to be $\mathcal{I}_{k}(S):=\operatorname{ker}\left(\rho_{k}\right)$ (and so with our indexing, which is different from some other authors, the classical Torelli group is $\mathcal{I}_{1}(S)$ ). To each $f \in \mathcal{I}_{k}=\mathcal{I}_{k}(S)$, we will associate an invariant $\Psi_{k}(f) \in \operatorname{End}\left(H_{1}(S, \mathbb{Z})\right)$ which is constructed from $\rho_{k+1}(f)$ (see below or Section 5). We will prove the following.

Theorem 1.1 (Criterion for pseudo-Anosovs) Let $f \in \mathcal{I}_{k}$. If the characteristic polynomial of $\Psi_{k}(f)$ is irreducible in $\mathbb{Z}[x]$, then $f$ is pseudo-Anosov.

Theorem 1.1 will follow immediately from the following theorem which we prove in Section 5. For the remainder of this paper, we let $H:=H_{1}(S, \mathbb{Z})$.

Theorem 1.2 Let $f \in \mathcal{I}_{k}$. If the characteristic polynomial $\chi\left(\Psi_{k}(f)\right)$ of $\Psi_{k}(f) \in$ $\operatorname{End}(H)$ has no (nontrivial) even degree or degree 1 factors over $\mathbb{Z}$, then $f$ is pseudoAnosov.

Since $\Psi_{k}$ uses only the data of $\rho_{k+1}(f)$ and $\operatorname{ker}\left(\rho_{k+1}\right)=\mathcal{I}_{k+1}$, we obtain the following corollary:

Corollary 1.3 If $f \in \mathcal{I}_{k}$ satisfies the hypothesis of Theorem 1.2, then the whole coset $f \mathcal{I}_{k+1}$ is pseudo-Anosov.

Note that the data of $\rho_{1}$ is not used in Theorem 1.2. Since

$$
\Gamma / \Gamma_{2}=H
$$

the homomorphism $\rho_{1}$ is the standard representation into $\operatorname{Aut}(H)$ with image isomorphic to the integral symplectic group $\operatorname{Sp}(2 g, \mathbb{Z})$. It is not too difficult to find a criterion on $\rho_{1}(f)$ for $f$ to be pseudo-Anosov, and in fact, Casson and Bleiler give such a criterion in [5, Lemma 5.1]. Casson and Bleiler show that if the characteristic polynomial, $\chi\left(\rho_{1}(f)\right)$, is irreducible over $\mathbb{Z}$, has no roots of unity as eigenvalues, and is not equal to $g\left(t^{n}\right)$ for any $n>1$ and $g \in \mathbb{Z}[x]$, then $f$ is pseudo-Anosov.

The Casson-Bleiler criterion is well-known and has been around for many years. It is unfortunately unable to detect pseudo-Anosovs in any of the $\mathcal{I}_{k}$ simply because 
$\mathcal{I}_{k} \subseteq \operatorname{ker}\left(\rho_{1}\right)$. (This is not to imply that the Casson-Bleiler criterion can detect all pseudo-Anosovs which act non-trivially on $H$; it cannot.) In this sense, this paper is an extension of the Casson-Bleiler criterion (for the case of a surface with one boundary component).

Remark 1.4 It is well-known that $\mathcal{I}_{1}$ has pseudo-Anosov elements thanks to criteria of Thurston [18], Penner [16], and others [2]. However, their methods of finding pseudo-Anosovs are all topological as opposed to algebraic in nature. Furthermore, their criteria require the specification of a particular mapping class and thus are not well-suited to dealing with the information of $\rho_{k}(f) \in \operatorname{Aut}\left(\Gamma_{1} / \Gamma_{k+1}\right)$ which only specifies a coset of $\mathcal{I}_{k}$. Both Thurston's criterion and Penner's criterion require that a mapping class be described in terms of twists about two multi-curves. In [2], BestvinaHandel describe an algorithm using train tracks that can determine whether any single mapping class is pseudo-Anosov or not. In fact, this algorithm has been implemented in a computer program by Peter Brinkmann [3].

Let us now outline the contents of the paper. In Section 2, we recall some basic properties of the series $\left\{\Gamma_{k}\right\}$. We then define for $f \in \mathcal{I}_{k}$ the invariant $\Psi_{k}(f) \in \operatorname{End}(H)$. $\left(\Psi_{k}(f)\right.$ is in general non-trivial which might be rather suprising given that $\rho_{1}(f) \in \operatorname{Aut}(H)$ is necessarily trivial.) To define $\Psi_{k}$, we need two ingredients, the Johnson homomorphism $\tau$ and contractions:

$$
\Phi_{2 k}: \Gamma_{2 k+1} / \Gamma_{2 k+2} \rightarrow H
$$

Defining $\Phi_{2 k}$ requires a bit of work and is described in Section 4. In Section 3, we recall the definition of the Johnson homomorphism $\tau$ which we describe here as follows:

$$
\tau: \mathcal{I}_{k}(S) \rightarrow \operatorname{Hom}\left(\bigoplus_{m=1}^{\infty} \Gamma_{m} / \Gamma_{m+1}, \bigoplus_{m=k+1}^{\infty} \Gamma_{m} / \Gamma_{m+1}\right)
$$

We denote the image of $f$ under $\tau$ as $\tau_{f}$. By the definition (given in Section 3), $\tau_{f}\left(\Gamma_{m} / \Gamma_{m+1}\right) \subseteq \Gamma_{m+k} / \Gamma_{m+k+1}$. We define $\Psi_{k}$ as follows:

$$
\Psi_{k}(f):=\left\{\begin{array}{ll}
\Phi_{k} \circ\left(\left.\tau_{f}\right|_{H}\right) & k \text { even } \\
\Phi_{2 k} \circ\left(\left.\tau_{f}^{2}\right|_{H}\right) & k \text { odd }
\end{array} \in \operatorname{End}(H)\right.
$$

Note that the map $\Psi_{k}$ is a homomorphism for $k$ even but not necessarily for $k$ odd.

In Section 5, we prove Theorem 1.2. The general idea of the proof of Theorem 1.2 is to use the Nielsen-Thurston classification which states that a mapping class is pseudo-Anosov if and only if it is neither reducible nor of finite order. Recall that $f$ is reducible if $f$ fixes the isotopy class of an essential 1-dimensional submanifold 
where essential means that each component is neither null-homotopic nor homotopic to a boundary component. Since $\mathcal{I}_{1}$ is torsion-free, the classification reduces to: $f$ is pseudo-Anosov if and only if it is irreducible. We then show that reducibility of $f$ implies that $\chi\left(\Psi_{k}(f)\right)$ has a linear or even degree factor by using the fact that a certain subgroup of $\pi_{1}(S)$ is invariant under $f_{*} \in \operatorname{Aut}\left(\pi_{1}(S)\right)$. The proof of Theorem 1.2 will be outlined in more detail in Section 5 .

For any particular $f \in \mathcal{I}_{k}$, the invariant $\Psi_{k}(f)$ is explicitly computable, provided one can compute $\tau_{f}$. In Section 6, we show some mapping classes satisfy the hypothesis of Theorem 1.2 by computing $\Psi_{k}(f)$ directly. Nevertheless, at present the author has not found whole families of pseudo-Anosovs ranging over either $g$ or $k$ which satisfy the hypothesis of Theorem 1.2. Additionally, in Section 6 we compare Theorem 1.2 to the Thurston/Penner criteria.

Remark 1.5 We choose to work with a surface with a boundary component as opposed to a closed surface to simplify things technically. The fundamental group of a surface with boundary is a free group. As we shall see in Section 2, this will further imply that the Lie algebra associated to the $\left\{\Gamma_{k}\right\}$ is a free Lie algebra. While the author suspects that one may obtain a criterion for closed surfaces from this criterion, he has not done so at present.

Acknowledgements The author would like to thank Dan Margalit, Nathan Broaddus, Ian Biringer, Juan Souto, Matthew Day and Asaf Hadari for their helpful comments. He would also like to thank Andy Putman for help during the research stage. He would like to especially thank Benson Farb for extensive comments, posing the question, continuous help and inspiration.

\section{Basic facts about the lower central series}

For the reader's convenience, we recall basic facts about central filtrations of a group. Suppose

$$
G=G_{1} \supset G_{2} \supset G_{3} \ldots
$$

is a filtration of $G$ by normal subgroups. We call $G$ a central filtration if $\left[G_{k}, G_{l}\right] \subseteq$ $G_{k+l}$. We recount the following folklore result.

Theorem 2.1 Let $\left\{G_{i}\right\}$ be a central filtration of $G$ by normal subgroups. Then, the following hold: 
(1) The function $G_{k} \times G_{l} \rightarrow G_{k+l}$ given by $(x, y) \mapsto x y x^{-1} y^{-1}$ induces a welldefined map

$$
G_{k} / G_{k+1} \times G_{l} / G_{l+1} \rightarrow G_{k+l} / G_{k+l+1}
$$

(2) Using the pairing from (1) as a bracket which we denote by [, ], we obtain a graded $\mathbb{Z}$-Lie algebra:

$$
L:=\bigoplus_{k} G_{k} / G_{k+1}
$$

For an explanation and proof see Sections 3.1 and 4.5 of Bass and Lubotzky [1]. Also, we recall for the reader that the lower central series is a central filtration (see $[1$, Section 4.4]).

The fundamental group of a surface with boundary is a free group. The Lie algebra associated to a free group's lower central series is a free Lie algebra [11, Theorem 5.12].

Theorem 2.2 Let $G$ be a free group with generators $a_{1}, \ldots, a_{n}$ and lower central series $G_{1} \supset G_{2} \supset \ldots$ Then the (graded) $\mathbb{Z}$-Lie algebra

$$
L:=\left(\bigoplus_{k} G_{k} / G_{k+1}, \quad[,]\right)
$$

is a free $\mathbb{Z}-$ Lie algebra. $L$ has as its generating set $\left\{a_{1}, \ldots, a_{n}\right\}$ viewed as a subset of $G_{1} / G_{2}$.

The definition of free Lie algebra is exactly what one expects: given a $\mathbb{Z}$-Lie algebra $L^{\prime}$ and elements $x_{1}, \ldots, x_{n} \in L^{\prime}$, there exists a unique Lie algebra homomorphism $h: L \rightarrow L^{\prime}$ such that $h\left(a_{i}\right)=x_{i}$. The free Lie algebra in general is fairly complicated. Even computing the rank of $G_{k} / G_{k+1}$ for arbitrary $k$ is nontrivial. Thankfully, free Lie algebras embed in simpler Lie algebras.

A free associative $\mathbb{Z}$-algebra $A$ with generators $b_{1}, \ldots, b_{n}$ is a noncommutative ring with the universal property that given a $\mathbb{Z}$-algebra $A^{\prime}$ and elements $x_{1}, \ldots, x_{n} \in A^{\prime}$ there is a unique homomorphism $h: A \rightarrow A^{\prime}$ such that $h\left(b_{i}\right)=x_{i}$. More concretely, $A$ is (canonically isomorphic to) the noncommutative polynomial ring in $n$ variables over $\mathbb{Z}$. However, viewing $A$ as a polynomial ring is not particularly convenient for the purposes of this paper. If we let $M:=\mathbb{Z}^{n}$, then $A$ is isomorphic to the tensor algebra $\bigoplus_{k=0}^{\infty} M^{\otimes k}$ where $M^{\otimes 0}:=\mathbb{Z}$. The algebra $A$ has a canonical Lie bracket: $[x, y]:=x \otimes y-y \otimes x$. Thus, we have a canonical Lie homomorphism $\mathcal{L} \rightarrow A$ defined by $a_{i} \mapsto b_{i}$. From Reutenauer [17, Corollary 0.3 and Theorem 0.5 ], we obtain the following. 
Theorem 2.3 If $L$ is a free $\mathbb{Z}$-Lie algebra with generators $a_{1}, \ldots, a_{n}$ and $A$ is a free associative algebra over $\mathbb{Z}$ with generators $b_{1}, \ldots, b_{n}$, then the canonical Lie homomorphism induced by $a_{i} \mapsto b_{i}$ is injective.

Moreover, it is not hard to check that the map $L \rightarrow A$ respects the grading.

Now, let us apply Theorems 2.2 and 2.3 to the group $\Gamma:=\pi_{1}(S)$ with (free) generators $a_{1}, \ldots, a_{2 g}$. Let $\mathcal{L}$ be the graded Lie algebra associated to $\left\{\Gamma_{k}\right\}$. Let $\mathcal{A}$ be the tensor algebra $\bigoplus_{k=0}^{\infty} H^{\otimes k}$ where $H^{\otimes 0}:=\mathbb{Z}$. Since $H \cong \mathbb{Z}^{2 g}$, the algebra $\mathcal{A}$ is a free associative algebra. To simplify notation, let us define $\mathcal{L}_{k}:=\Gamma_{k} / \Gamma_{k+1}$. Recall that $\mathcal{A} \cong \bigoplus_{k=0}^{\infty} M^{\otimes k}$ where $M=\mathbb{Z}^{2 g}$. We have defined the $a_{i}$ as elements of $\pi_{1}(S)$, but we can also consider the equivalence class of $a_{i}$ in $\Gamma_{1} / \Gamma_{2} \subset \mathcal{L}$ or in $H=H^{\otimes 1} \subset \mathcal{A}$. Thus, we obtain a natural, injective map $\mathcal{L} \rightarrow \mathcal{A}$ defined by sending " $a_{i}$ " to " $a_{i}$ ".

The mapping class group has a natural action on $\mathcal{L}$ by considering

$$
\mathcal{L}=\bigoplus_{k=1}^{\infty} \Gamma_{k} / \Gamma_{k+1}
$$

as a direct sum of representations $\operatorname{Mod}(S) \rightarrow \operatorname{Aut}\left(\Gamma_{k} / \Gamma_{k+1}\right)$. We obtain an action on

$$
\mathcal{A}=\bigoplus_{k=0}^{\infty} H^{\otimes k}
$$

from the action on $H$. It is not hard to check that the map $\mathcal{L} \rightarrow \mathcal{A}$ respects this action. Since the $\operatorname{Mod}(S)$-action on $\mathcal{A}$ is induced by the action on $H$, it factors through to an $\operatorname{Sp}(2 g, \mathbb{Z})$-action and so the $\operatorname{Mod}(S)$-action on $\mathcal{L}$ factors through $\operatorname{Sp}(2 g, \mathbb{Z})$ also (This can also be proven directly.).

\section{The Johnson Homomorphisms}

All of the results in this section are the work of Johnson, Morita, Hain and others. Recall that

$$
\mathcal{I}_{k}:=\operatorname{ker}\left(\operatorname{Mod}(S) \rightarrow \operatorname{Aut}\left(\Gamma_{1} / \Gamma_{k+1}\right)\right)
$$

and $H=H_{1}(S)$. A preliminary version of the Johnson homomorphism is a map:

$$
\tau: \mathcal{I}_{k} \rightarrow \operatorname{Hom}\left(H, \Gamma_{k+1} / \Gamma_{k+2}\right)
$$

for each $k$. Note that the image of $f$ under $\tau$ will be denoted $\tau_{f}$ as is standard. We define the preliminary version as follows. Let $f \in \mathcal{I}_{k}$. Since $f_{*}$ acts trivially on 
$\Gamma_{1} / \Gamma_{k+1}$, we obtain a well-defined map of sets:

$$
\begin{aligned}
t_{f}: \Gamma_{1} / \Gamma_{k+2} & \rightarrow \Gamma_{k+1} / \Gamma_{k+2} \\
x & \mapsto f_{*}(x) x^{-1}
\end{aligned}
$$

The following result is one part of [14, Proposition 2.3].

Proposition 3.1 (Johnson, Morita) The set map $t_{f}: \Gamma_{1} / \Gamma_{k+2} \rightarrow \Gamma_{k+1} / \Gamma_{k+2}$ induces a well-defined homomorphism $H \rightarrow \Gamma_{k+1} / \Gamma_{k+2}$ which is $\tau_{f}$. Moreover, $\tau$ is a homomorphism.

Proof By the very definition of the lower central series, $\Gamma_{k+1} / \Gamma_{k+2}$ is in the center of $\Gamma_{1} / \Gamma_{k+2}$. Thus,

$$
f_{*}(x y)(x y)^{-1}=f_{*}(x y) y^{-1} x^{-1}=f_{*}(x)\left(f_{*}(y) y^{-1}\right) x^{-1}=f_{*}(x) x^{-1}\left(f_{*}(y) y^{-1}\right)
$$

and so $t_{f}$ is in fact a homomorphism. As $\Gamma_{k+1} / \Gamma_{k+2}$ is abelian, this homomorphism factors through the abelianization of $\Gamma_{1} / \Gamma_{k+1}$ which is $\Gamma_{1} /\left[\Gamma_{1}, \Gamma_{1}\right]=\Gamma_{1} / \Gamma_{2}=H$. Hence, we obtain a homomorphism $H \rightarrow \Gamma_{k+1} / \Gamma_{k+2}$. Now, suppose we are given $f, g \in \mathcal{I}_{k}$. Then, we have

$$
\begin{gathered}
f_{*}\left(g_{*}(x)\right) x^{-1}=f_{*}\left(g_{*}(x) x^{-1}\right) f_{*}(x) x^{-1} \\
=\left(f_{*}\left(t_{g}(x)\right) t_{g}(x)^{-1}\right) t_{g}(x) f_{*}(x) x^{-1}=t_{f}\left(t_{g}(x)\right) t_{g}(x) t_{f}(x)
\end{gathered}
$$

Since $t_{g}(x) \in \Gamma_{k+1} / \Gamma_{k+2} \subseteq \operatorname{ker} t_{f}$, we find that $f_{*}\left(g_{*}(x)\right) x^{-1}=t_{g}(x) t_{f}(x)$.

Remark 3.2 In the above proof, we see that $\operatorname{ker}\left(t_{f}\right) \supset \Gamma_{2} / \Gamma_{k+2}$, and so for $x \in$ $\Gamma_{2} / \Gamma_{k+2}$ we have

$$
1=t_{f}(x)=f_{*}(x) x^{-1} \Rightarrow f(x)=x
$$

Thus $f$ acts trivially on $\Gamma_{2} / \Gamma_{k+2}$ and in particular on $\Gamma_{k+1} / \Gamma_{k+2}$. Looking at the short exact sequence

$$
1 \rightarrow \Gamma_{k+1} / \Gamma_{k+2} \rightarrow \Gamma_{1} / \Gamma_{k+2} \rightarrow \Gamma_{1} / \Gamma_{k+1} \rightarrow 1
$$

one might think that $f$ must act trivially on $\Gamma_{1} / \Gamma_{k+2}$ itself, but this is not the case. Elements in $\left(\Gamma_{1} / \Gamma_{k+2}\right) \backslash\left(\Gamma_{2} / \Gamma_{k+2}\right)$ may be changed by elements in $\Gamma_{k+1} / \Gamma_{k+2}$ and this is precisely what $\tau_{f}$ measures.

In view of the remark, we see that $\tau_{f}$ retains the information of $f_{*} \in \operatorname{Aut}\left(\Gamma_{1} / \Gamma_{k+2}\right)$. Furthermore, $\tau_{f}$ determines $f_{*}$ as an element of $\operatorname{Aut}\left(\Gamma_{1} / \Gamma_{k+2}\right)$ (assuming $f \in \mathcal{I}_{k}$ ). We simply note that $f_{*}(x)=\tau_{f}(\bar{x}) x$ where $\bar{x}$ is the projection of $x$ to $H$. Moreover, the following sequence is exact [14, Proposition 2.3]:

$$
1 \rightarrow \operatorname{Hom}\left(H, \Gamma_{k+1} / \Gamma_{k+2}\right) \rightarrow \operatorname{Aut}\left(\Gamma_{1} / \Gamma_{k+2}\right) \rightarrow \operatorname{Aut}\left(\Gamma_{1} / \Gamma_{k+1}\right)
$$


Given $f \in \mathcal{I}_{k}$, one can similarly define a function:

$$
\begin{aligned}
\Gamma_{m} / \Gamma_{m+k+1} & \rightarrow \Gamma_{m+k} / \Gamma_{m+k+1} \\
x & \mapsto f_{*}(x) x^{-1}
\end{aligned}
$$

As before, this induces a well-defined homomorphism $\Gamma_{m} / \Gamma_{m+1} \rightarrow \Gamma_{m+k} / \Gamma_{m+k+1}$. (See [12, Lemma 3.2].)

Consider the free associative algebra $\mathcal{A}$ as defined in the previous section. Suppose one has chosen $2 g$ elements $\left\{x_{1}, \ldots, x_{2 g}\right\} \subseteq \mathcal{A}$. From general theory about the free associative algebra, we know there is then a unique derivation $D: \mathcal{A} \rightarrow \mathcal{A}$ such that $D\left(a_{i}\right)=x_{i}$ where the $a_{i}$ are generators of $\mathcal{A}$ (see [17, Lemma 0.7]). If $x_{i} \in \mathcal{L}$, then induction on the grading and the following computation show that $D(\mathcal{L}) \subseteq \mathcal{L}$ and that $D$ is a derivation on $\mathcal{L}$ :

$$
D[y, z]=D(y z-z y)=(D y) z+y D z-(D z) y-z D y=[D y, z]+[y, D z]
$$

Thus, given $f \in \mathcal{I}_{k}$, there is a unique derivation $D_{f}$ of $\mathcal{A}$ which extends $\tau_{f}$. It turns out that extending $\tau_{f}$ to all of $\mathcal{L}$ yields the same result regardless of whether one restricts $D_{f}$ or uses (3). The following proposition follows more or less from [12, Lemma 2.3 and Proposition 2.5].

Proposition 3.3 (Morita) For all $m \geq 1$, the map defined by (3) induces a homomorphism $\Gamma_{m} / \Gamma_{m+1} \rightarrow \Gamma_{m+k} / \Gamma_{m+k+1}$ and is equal to the map $\left.D_{f}\right|_{\mathcal{L}_{m}}$.

By abuse of notation, we will denote the extention to $\mathcal{L}$ by $\tau_{f}$. The map $\tau$ has other nice algebraic properties. They are collected in the following theorem.

Theorem 3.4 (Morita) Let $\tau$ be as defined above, a collection of homomorphisms $\mathcal{I}_{k} \rightarrow \operatorname{Der}(\mathcal{L})$, one for each $k$. Then, the following hold:

(a) The map $\tau: \mathcal{I}_{k} \rightarrow \operatorname{Der}(\mathcal{L})$ is a homomorphism with kernel $\mathcal{I}_{k+1}$. Hence, it induces a well-defined homomorphism $\mathcal{I}_{k} / \mathcal{I}_{k+1} \rightarrow \operatorname{Der}(\mathcal{L})$.

(b) The abelian group

$$
\bigoplus_{k=1}^{\infty} \mathcal{I}_{k} / \mathcal{I}_{k+1}
$$

has a Lie algebra structure induced by:

$$
\begin{aligned}
\mathcal{I}_{m} \times \mathcal{I}_{n} & \rightarrow \mathcal{I}_{m+n} \\
(f, g) & \mapsto f g f^{-1} g^{-1}=:[f, g]
\end{aligned}
$$


(c) The map $\tau$ induces a Lie algebra homomorphism:

$$
\bigoplus_{k=1}^{\infty} \mathcal{I}_{k} / \mathcal{I}_{k+1} \rightarrow \operatorname{Der}(\mathcal{L})
$$

Furthermore, $\tau$ respects the conjugation action of $\operatorname{Mod}(S)$ on $\mathcal{I}_{k}$ and $\operatorname{Der}(\mathcal{L})$.

Sketch of proof This proof sketch will consist mainly of citations. For (a), recall that by Proposition 3.1, $\left.\tau_{f \circ g}\right|_{H}=\left.\tau_{f}\right|_{H}+\left.\tau_{g}\right|_{H}$. Since the derivations $\tau_{f \circ g}$ and $\tau_{f}+\tau_{g}$ agree on generators, they must agree on all of $\mathcal{L}$. One deduces the kernel is $\mathcal{I}_{k+1}(S)$ from the exact sequence in (2). Part (b) is [13, Proposition 4.1]. Also, [13, Proposition 4.7] shows (in slightly different notation) that $\left.\tau_{[f, g]}\right|_{H}=\left.\left(\tau_{f} \tau_{g}-\tau_{g} \tau_{f}\right)\right|_{H}$. Since the two derivations $\tau_{[f, g]}$ and $\tau_{f} \tau_{g}-\tau_{g} \tau_{f}$ agree on $H$ and since $H$ generates $\mathcal{L}$, we must have equality. To show that the $\operatorname{Mod}(S)$ action is respected, we use the definition of $\tau_{f}$ given by (3). Suppose $g \in \operatorname{Mod}(S)$. In $\Gamma_{m} / \Gamma_{m+k+1}$, we have:

$$
\begin{aligned}
\tau_{g f g^{-1}}(x) & =g\left(f\left(g^{-1}(x)\right)\right) x^{-1}=g\left(f(g(x)) g^{-1}\left(x^{-1}\right)\right) \\
& =g\left(f\left(g^{-1}(x)\right)\left(g^{-1}(x)\right)^{-1}\right)=g\left(\tau_{f}\left(g^{-1}(x)\right)\right)
\end{aligned}
$$

Remark 3.5 A priori, it may seem that, for $f \in \mathcal{I}_{k}$, we are using the entire action of $f_{*}$ on $\pi_{1}(S)$ since we use the action on $\Gamma_{m} / \Gamma_{m+k+1}$ for all $m$. This would conflict with the characterization given in the introduction that we only use the data of $f_{*} \in \operatorname{Aut}\left(\Gamma_{1} / \Gamma_{k+2}\right)$. However, since $\tau_{f}$ is a derivation on $\mathcal{L}$ which is generated by $H$, it is completely determined by $\left.\tau_{f}\right|_{H}$ which is itself determined by $f_{*} \in \operatorname{Aut}\left(\Gamma_{1} / \Gamma_{k+2}\right)$.

\section{The contractions $\Phi_{k}$}

Our goal in this section is to find a contraction $\mathcal{L}_{k+1} \rightarrow \mathcal{L}_{1}$ respecting the Sp-action and thus the $\operatorname{Mod}(S)$-action by the results of Section 2 . We remark that we want to respect the action so that $\chi\left(\Psi_{k}(f)\right)$ will depend only on the conjugacy class of $f$ and because the argument in Section 5 implicitly uses a change of coordinates. The following theorem simplifies this problem. Below, Hom $\mathrm{Sp}$ will denote the set of homomorphisms which respect the $\mathrm{Sp}$ action, and, for $X$ an Sp-representation, $X_{\mathrm{Sp}}$ will indicate the space of vectors fixed by the Sp action. While I suspect the following may be known, I was not able to find it in the literature.

Theorem 4.1 If $f \in \operatorname{Hom}_{\mathrm{Sp}}\left(\mathcal{L}_{k+1}, \mathcal{L}_{1}\right)$, then $\exists n \in \mathbf{Z}$ such that $n f$ is the restriction of an element $g \in \operatorname{Hom}_{\mathrm{Sp}}\left(\mathcal{A}_{k+1}, \mathcal{A}_{1}\right)$, where $\mathcal{A}_{m}$ is the summand $H^{\otimes m} \subset \mathcal{A}$. 
Proof The theorem will follow if we can find a bilinear pairing on each $\mathcal{A}_{k+1}$ which is nondegenerate on both $\mathcal{A}_{k+1}$ and $\mathcal{L}_{k+1}$. Let $\left\{a_{1}, b_{1}, \ldots, a_{g}, b_{g}\right\}$ be a symplectic basis of $H_{1}(S)$. The $a_{i}$ and $b_{i}$ also serve as a free generating set of $\mathcal{L}$ as a Lie algebra and of $\mathcal{A}$ as an associative algebra. We can easily define a pairing $\langle$,$\rangle which$ is nondegenerate on $\mathcal{A}_{k+1}$. If $x=x_{1} \otimes x_{2} \cdots \otimes x_{k+1}$ and $y=y_{1} \otimes y_{2} \cdots \otimes y_{k+1}$, then set

$$
\langle x, y\rangle:=\left\langle x_{1}, y_{1}\right\rangle\left\langle x_{2}, y_{2}\right\rangle \ldots\left\langle x_{k+1}, y_{k+1}\right\rangle
$$

where $\left\langle x_{i}, y_{i}\right\rangle$ is the algebraic intersection pairing on $H$.

Now, let $\theta \in \operatorname{Aut}(\mathcal{A})$ be the algebra homomorphism defined by $\theta\left(a_{i}\right)=b_{i}$ and $\theta\left(b_{i}\right)=$ $-a_{i}$. In particular, if $w=x_{1} \otimes x_{2} \cdots \otimes x_{n}$ then $\theta(w)=\theta\left(x_{1}\right) \otimes \theta\left(x_{2}\right) \otimes \cdots \otimes \theta\left(x_{n}\right)$. Let $Y_{k}$ be the canonical basis of $H^{\otimes k}$ induced by the basis of $H$ (ie, tensoring the $a$ 's and $b$ 's in every possible order). For two elements $y, y^{\prime} \in Y_{k}$, one easily sees that $\left\langle y, y^{\prime}\right\rangle \neq 0$ if and only if $y^{\prime}= \pm \theta(y)$. Then, for $P=\sum_{y} c_{y} y$, we have $\langle P, \theta(P)\rangle>0$, since all "cross terms" vanish and we are left with $\sum_{y} c_{y}^{2}\langle y, \theta(P)\rangle$.

We now wish to show that $\langle$,$\rangle is nondegenerate on the embedded copy of \mathcal{L}_{k+1}$, but this is almost immediate. We only need that $P \in \mathcal{L}_{k+1}$ implies $\theta(P) \in \mathcal{L}_{k+1}$. Indeed, since $\mathcal{L}$ is the Lie subalgebra of $\mathcal{A}$ generated by $\left\{a_{1}, b_{1}, \ldots, a_{g}, b_{g}\right\}$ and since $\theta$ preserves the Lie bracket and (up to sign) permutes the generators $\left\{a_{1}, b_{1}, \ldots, a_{g}, b_{g}\right\}$, we see that $\theta(\mathcal{L})=\mathcal{L}$.

Suppose $f \in \operatorname{Hom}_{\mathrm{Sp}}\left(\mathcal{L}_{k+1}, \mathcal{L}_{1}\right) \cong\left(\mathcal{L}_{k+1}^{*} \otimes \mathcal{L}_{1}\right)_{\mathrm{Sp}}$. Since $\mathcal{L}_{k+1}$ and $\mathcal{L}_{k+1}^{*}$ are finitely generated free $\mathbf{Z}$-modules, the pairing $\langle$, $\rangle$ gives an embedding $\mathcal{L}_{k+1} \hookrightarrow \mathcal{L}_{k+1}^{*}$ whose image has finite index. Thus, there is some $n \in \mathbf{Z}$ such that $n f$ is in the image of $\left(\mathcal{L}_{k+1} \otimes \mathcal{L}_{1}\right)_{\mathrm{Sp}}$, but we have:

$$
\left(\mathcal{L}_{k+1} \otimes \mathcal{L}_{1}\right)_{\mathrm{Sp}} \hookrightarrow\left(\mathcal{A}_{k+1} \otimes \mathcal{A}_{1}\right)_{\mathrm{Sp}} \hookrightarrow\left(\mathcal{A}_{k+1}^{*} \otimes \mathcal{A}_{1}\right)_{\mathrm{Sp}} \cong \operatorname{Hom}_{\mathrm{Sp}}\left(\mathcal{A}_{k+1}, \mathcal{A}_{1}\right)
$$

Thus, $n f$ is the restriction of some $g \in \operatorname{Hom}_{\mathrm{Sp}}\left(\mathcal{A}_{k+1}, \mathcal{A}_{1}\right)$.

Theorem 4.1 and its proof reduce our problem to finding tensors in $\left(\mathcal{A}_{k+1} \otimes \mathcal{A}_{1}\right)_{\mathrm{Sp}} \cong$ $\left(H^{\otimes k+2}\right)_{\text {Sp }}$. Thus, if $k=2 n$ is even, we obtain such a tensor by taking the symplectic pairing $\omega_{0}=\sum_{i}\left(a_{i} \otimes b_{i}-b_{i} \otimes a_{i}\right)$ and taking high tensor powers, ie, $\omega_{0}^{\otimes(n+1)}$. The element $\omega_{0}^{\otimes(n+1)}$ represents the contraction

$$
x_{1} \otimes x_{2} \otimes \cdots \otimes x_{k+1} \mapsto\left(\prod_{j=1}^{n}\left\langle x_{2 j-1}, x_{2 j}\right\rangle\right) x_{k+1} .
$$

This contraction is what we denote by $\Phi_{k}$. 
There is an obvious action of the permutation group $\mathfrak{S}_{2 m}$ on $H^{\otimes 2 m}$. Since $\operatorname{Sp}(2 g, \mathbb{Z})$ acts diagonally on $H^{\otimes 2 m}$, it is easy to see that for any $\sigma \in \mathfrak{S}_{2 m}$, we have $\eta \in\left(H^{\otimes 2 m}\right)_{\mathrm{Sp}}$ if and only if $\sigma(\eta) \in\left(H^{\otimes 2 m}\right)_{\mathrm{Sp}}$. Thus, all the vectors $\sigma\left(\omega_{0}^{\otimes 2 m}\right)$ are Sp-invariant as well. For every $\sigma \in \mathfrak{S}_{2 m}$, there is a corresponding $\sigma^{\prime}$ so that $\sigma\left(\omega_{0}^{2 m}\right)$ corresponds to the contraction:

$$
x_{1} \otimes x_{2} \otimes \cdots \otimes x_{m-1} \mapsto\left(\prod_{j=1}^{n}\left\langle x_{\sigma^{\prime}(2 j-1)}, x_{\sigma^{\prime}(2 j)}\right\rangle\right) x_{\sigma^{\prime}(m-1)}
$$

Furthermore, it is a classical result of Weyl (see for example, [15, Section 4.1]) that $\left\{\sigma\left(\omega_{0}^{\otimes 2 m}\right)\right\}_{\sigma \in \mathfrak{S}_{2 m}}$ is a generating set for $\left((H \otimes \mathbb{Q})^{\otimes 2 m}\right)_{\operatorname{Sp}(2 g, \mathbb{Q})}$. By a result of Borel (see [4, Theorem 2.7 and Remark 2.8]), we can conclude that all $\operatorname{Sp}(2 g, \mathbb{Z})$-fixed vectors are $\operatorname{Sp}(2 g, \mathbb{Q})$-fixed vectors and thus $\left(H^{\otimes 2 m}\right)_{\operatorname{Sp}(2 g, \mathbb{Z})}=\left\langle\sigma\left(\omega_{0}^{\otimes 2 m}\right) \mid \sigma \in \mathfrak{S}_{2 m}\right\rangle$.

\section{Proof of Theorem 1.2}

Recall from above that for each $k \geq 1$ we defined a map:

$$
\begin{aligned}
\Psi_{k}: \mathcal{I}_{k} & \rightarrow \operatorname{End}(H) \\
f & \mapsto \begin{cases}\Phi_{k} \circ\left(\left.\tau_{f}\right|_{H}\right) & k \text { even } \\
\Phi_{2 k} \circ\left(\left.\tau_{f}^{2}\right|_{H}\right) & k \text { odd }\end{cases}
\end{aligned}
$$

Idea of proof of Theorem 1.2 Before diving into the proof, let us sketch the idea of the proof for one of the main cases. The goal is to show that if $f \in \mathcal{I}_{k}$ fixes the isotopy class of some curve $\gamma$, then $\Psi_{k}(f)$ has a non-trivial invariant subspace in $H$. Let us look at the case where $f$ fixes some separating curve $\gamma$ in $S$. If we were trying to prove Casson-Bleiler in this case, we would note that $f$ leaves two subspaces of $H$ invariant which correspond to the two invariant subsurfaces, call them $S_{1}$ and $S_{2}$ (See Figure 1.) Now suppose $f \in \mathcal{I}_{2}$ and the elements $c_{i}$ (resp $d_{i}$ ) generate the image $C$ of $H_{1}\left(S_{1}, \mathbb{Z}\right)$ (resp. the image $D$ of $H_{1}\left(S_{2}, \mathbb{Z}\right)$ ) in $H$. Since $\hat{\imath}\left(c_{i}, d_{j}\right)=0$ for all $i, j$ and $\Phi_{k}$ is defined via $\hat{\imath}$, one might have the hope that $\tau_{f}(C) \subseteq\left\langle\left[c_{i},\left[c_{j}, c_{k}\right]\right]\right|$ all $\left.i, j, k\right\rangle$ and $\tau_{f}(D) \subseteq\left\langle\left[d_{i},\left[d_{j}, d_{k}\right]\right]\right|$ all $\left.i, j, k\right\rangle$ so as to obtain that $\Psi_{2}(f)(C) \subseteq C$ and $\Psi_{2}(f)(D) \subseteq D$.

The hope that $\tau_{f}(C) \subseteq\left\langle\left\{\left[c_{i},\left[c_{j}, c_{k}\right]\right]\right\}\right\rangle$ and $\tau_{f}(D) \subseteq\left\langle\left\{\left[d_{i},\left[d_{j}, d_{k}\right]\right]\right\}\right\rangle$ is, in fact, false, but the degree to which this naive hope fails can be controlled. What one can say is that (if we take $S_{2}$ as in Figure 1) $\pi_{1}\left(S_{2}\right)$ is an $f$-invariant subgroup of $\pi_{1}(S)$. Since $\pi_{1}\left(S_{2}\right)$ can be generated by lifts of the $d_{i}$ and of $\gamma$ and since $\gamma \in\left\langle\left[\tilde{c_{i}}, \widetilde{c_{j}}\right]\right|$ all $\left.i, j\right\rangle$ for some lifts $\widetilde{c_{i}} \in \pi_{1}(S)$ of the $c_{i}$, it turns out that $\tau_{f}\left(d_{i}\right) \in$ $\left\langle\left[d_{i},\left[d_{j}, d_{k}\right]\right],\left[d_{i},\left[c_{j}, c_{k}\right]\right]\right|$ all $\left.i, j, k\right\rangle$. It is then not to hard to see that $\Phi_{2}\left(\tau_{f}\left(d_{i}\right)\right) \subseteq$ 
$\left\langle\left\{d_{i}\right\}\right\rangle=D$, and so $\Psi_{2}(f)$ has an invariant subspace $D$. The full proof of the separating case is a more general (and complete) version of this last argument.

We remark that the following proof of the main theorem remains valid if we replace $\Phi_{k}$ with any of the contractions induced by a $\sigma\left(\omega_{0}^{k+2}\right)$ described in Section 4 . In the following, all factorization and irreducibility is with respect to $\mathbb{Z}[x]$.

Proof of Theorem 1.2 Now let us prove the theorem. Let $f \in \mathcal{I}_{k}$. Recall that the Nielsen-Thurston classification and torsion-freeness of $\mathcal{I}_{1} \supseteq \mathcal{I}_{k}$ imply that $f$ is pseudo-Anosov if and only if $f$ is irreducible. It is well-known that $\mathcal{I}_{1}$ is pure, meaning that if an isotopy class of 1-submanifold is fixed, then each component of the 1-submanifold is fixed (see Ivanov [9, Theorem 1.2]). Thus, the proof of Theorem 1.2 reduces to proving the following two claims.

Claim 1 Suppose $f$ fixes an essential separating curve. Then, the characteristic polynomial of $\Psi_{k}(f)$ factors into two (nontrivial) even degree polynomials in $\mathbb{Z}[x]$.

Claim 2 Suppose $f$ fixes a nonseparating curve. Then, $\Psi_{k}(f)$ has an eigenvector over $\mathbb{Z}$.

Before we begin the proofs of Claims 1 and 2, we state a theorem that will be used for both. (This is [17, Theorem 2.5])

Theorem 5.1 (Shirshov, Witt) If $\mathcal{L}^{\prime}$ is a subalgebra of a free Lie algebra $\mathcal{L}$ over a field, then $\mathcal{L}^{\prime}$ is a free Lie algebra .

Proof of Claim 1 Let $\gamma$ be the (oriented) separating curve such that $f(\gamma)=\gamma$. Cutting along $\gamma$ separates $S$ into a $\Sigma_{g_{1}, 1}=: S_{1}$ and a $\Sigma_{g_{2}, 2}=: S_{2}$ where $g_{1}+g_{2}=g$. Let $C$ (resp. $D)$ be the image of $H_{1}\left(S_{1}, \mathbb{Z}\right)$ (resp. $\left.H_{1}\left(S_{2}, \mathbb{Z}\right)\right)$ in $H$. Since $f\left(S_{i}\right)=S_{i}$ (up to isotopy), one might hope that either $\Psi_{k}(f)(C) \subseteq C$ or $\Psi_{k}(f)(D) \subseteq D$. We will show that this actually holds for $D$.

We begin by defining a submodule of $\mathcal{L}$ :

$$
M:=\bigoplus_{m}\left(\Lambda \cap \Gamma_{m} / \Lambda \cap \Gamma_{m+1}\right)
$$

where $\Lambda:=\pi_{1}\left(S_{2}\right)$. Note that $M \cap \mathcal{L}_{1}=D$. Step 1 is to show that $\tau_{f}(M) \subseteq M$. Step 2 is to show that $M$ is a free Lie subalgebra and give generators of $M$ as a Lie algebra. Step 3 is to show, using the generators, that for any $x \in M$ we have $\Phi_{n}(x) \in D$. Then, it is clear from the definition of $\Psi_{k}$ that for $d \in D$, we have $\Psi_{k}(f)(d) \in D$. Since $D$ is an even rank subspace, that will complete the proof. 
First, we need to set up some notation. Let $p_{1} \in \partial S_{1}$ (resp. $p_{2} \in \partial S_{2} \cap \partial S$ ) be the basepoint of $S_{1}$ (resp $S_{2}$ and $S$ ). Let $\alpha$ be a path from $p_{2}$ to $p_{1}$, and let $\tilde{\gamma}=\alpha \gamma \alpha^{-1} \in \pi_{1}\left(S_{2}\right)$. Let $\iota$ (resp. $\hat{\iota}$ ) denote geometric (resp. algebraic) intersection number of unbased homotopy classes of closed curves. Choose $\left\{c_{i}^{\prime}\right\}_{i=1}^{2 g_{1}} \in \pi_{1}\left(S_{1}, p_{1}\right)$ and $\left\{d_{i}\right\}_{i=1}^{2\left(g_{2}\right)} \in \pi_{1}\left(S_{1}, p_{2}\right)$ with the following properties (see Figure 1):

(a) The set $\left\{c_{i}^{\prime}\right\}_{i=1}^{2 g_{1}}\left(\right.$ resp. $\left.\{\tilde{\gamma}\} \cup\left\{d_{i}\right\}_{i=1}^{2\left(g_{2}\right)}\right)$ generates $\pi_{1}\left(S_{1}, p_{1}\right)$ (resp. $\left.\pi_{1}\left(S_{2}, p_{2}\right)\right)$.

(b) For all $m, n$, we have $\iota\left(c_{m}^{\prime}, d_{n}\right)=\widehat{\imath}\left(c_{m}^{\prime}, d_{n}\right)=0$. Furthermore,

$$
\begin{aligned}
& \iota\left(c_{m}^{\prime}, c_{n}^{\prime}\right)= \begin{cases}1 & \text { if } m=n+g_{1} \text { or } m=n-g_{1} \\
0 & \text { otherwise }\end{cases} \\
& \iota\left(d_{m}, d_{n}\right)= \begin{cases}1 & \text { if } m=n+g_{2} \text { or } m=n-g_{2} \\
0 & \text { otherwise }\end{cases}
\end{aligned}
$$

and for $1 \leq i \leq g_{1}$ (resp. $\left.1 \leq i \leq g_{2}\right)$, we have $\hat{\imath}\left(c_{i}^{\prime}, c_{i+g_{1}}^{\prime}\right)=1$ (resp. $\left.\widehat{\iota}\left(d_{i}, d_{i+\left(g_{2}\right)}\right)=1\right)$.

(c) As an element of $\pi_{1}\left(S_{1}, p_{1}\right)$, we have $\gamma=\prod_{i=1}^{g_{1}}\left[c_{i}^{\prime}, c_{i+g_{1}}^{\prime}\right]$.

In particular, the union $\left\{c_{i}^{\prime}\right\}_{i=1}^{2 g_{1}} \cup\left\{d_{i}\right\}_{i=1}^{2 g_{2}}$ gives a symplectic basis in $H$. Now, let $c_{i}$ := $\alpha c_{i}^{\prime} \alpha^{-1}$. We have $\tilde{\gamma}=\prod_{i=1}^{g_{1}}\left[c_{i}, c_{i+g_{1}}\right]$ and $\pi_{1}\left(S, p_{2}\right)=\left\langle\left\{c_{i}\right\},\left\{d_{i}\right\}\right\rangle$. Furthermore, denote the inclusion map of $S_{2}$ by $j: S_{2} \hookrightarrow S$. In the following, we will frequently view $d_{i} \in \mathcal{L}_{1}$ and $\tilde{\gamma} \in \mathcal{L}_{2}$.

$S_{1}$

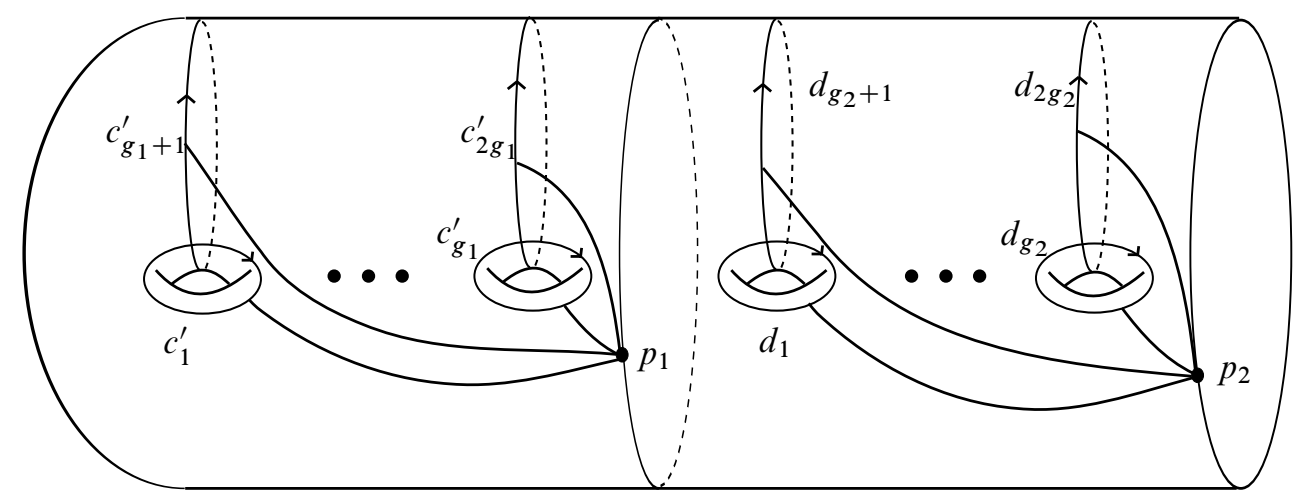

Figure 1

Step 1 First note that since $S$ and $S_{2}$ share a base point, $\pi_{1}\left(S_{2}\right)$ gives a well-defined subgroup of $\pi_{1}(S)=\Gamma$ which is invariant under $f_{*}$. We remark that a similar statement 
is not true for $S_{1}$. Indeed, to embed $\pi_{1}\left(S_{1}\right)$ in $\pi_{1}(S)$ requires that we choose a path connecting base points (eg $\alpha$ ); even after choosing a representative homeomorphism of $f$ which fixes $\gamma$ pointwise, this path is not necessarily preserved (up to homotopy rel endpoints).

Recall that one way of defining $\tau_{f}$ is to induce it from the map:

$$
\begin{aligned}
\Gamma_{m} & \rightarrow \Gamma_{m+k} \\
x & \mapsto f_{*}(x) x^{-1}
\end{aligned}
$$

Since $f_{*}(\Lambda)=\Lambda$, it is easy to see that

$$
M=\bigoplus_{m}\left(\Lambda \cap \Gamma_{m} / \Lambda \cap \Gamma_{m+1}\right)
$$

is a $\tau_{f}$-invariant submodule of $\mathcal{L}$.

Step 2 We wish to show $M$ is a Lie subalgebra and find its generators. We will do this by showing that $M$ is the Lie algebra homomorphic image of a Lie algebra $N$ whose generators are easily found.

We first define a filtration of $\Lambda$ which is a slight alteration of the lower central series. We let:

$$
\begin{aligned}
\Lambda_{1} & :=\pi_{1}\left(S_{2}\right) \\
\Lambda_{2} & :=\left\langle\left[\Lambda_{1}, \Lambda_{1}\right], \tilde{\gamma}\right\rangle \\
\Lambda_{m} & :=\left\langle\left[\Lambda_{m-n}, \Lambda_{n}\right]\right\rangle_{n=1}^{\left\lfloor\frac{m}{2}\right\rfloor} \text { for } m \geq 3
\end{aligned}
$$

By Theorem 2.1,

$$
N:=\bigoplus_{n} \Lambda_{n} / \Lambda_{n+1}
$$

is a graded $\mathbb{Z}$-Lie algebra under the commutation bracket. Since $j_{*}\left(\Lambda_{n}\right) \subseteq \Gamma_{n}$, there is an induced Lie algebra homomorphism $N \rightarrow \mathcal{L}$. It is easy to check that, as a Lie algebra, $N$ is generated by $\left\{d_{i}\right\}_{i=1}^{2 g_{2}} \cup\{\tilde{\gamma}\}$ and so its image $M^{\prime}:=j_{*}(N)$ in $\mathcal{L}$ is also generated by $\left\{d_{i}\right\}_{i=1}^{2 g_{2}} \cup\{\tilde{\gamma}\}$ (viewed in $\mathcal{L}$ ).

Proposition 5.2 $N$ maps isomorphically onto $M^{\prime}$

Proof of Proposition 5.2 We wish to use Theorem 5.1, but $\mathcal{L}$ is not an algebra over a field. As $\mathbb{Q}$ is a flat $\mathbb{Z}$-module, we have $M^{\prime} \otimes \mathbb{Q} \hookrightarrow \mathcal{L} \otimes \mathbb{Q}$, and so $M_{\mathbb{Q}}^{\prime}:=M^{\prime} \otimes \mathbb{Q}$ is a free Lie algebra generated by $\left\{d_{i}\right\}_{i=1}^{2\left(g_{2}\right)} \cup\{\tilde{\gamma}\}$, but it is not a priori clear that these generators are free. In the proof of Theorem 5.1 in [17], a recipe is given for finding free generators of a subalgebra, which we describe now.

Algebraic ${ }^{3} \mathcal{G}$ Geometric Topology, Volume 7 (2007) 
For any subset $X \subseteq \mathcal{L} \otimes \mathbb{Q}$, let $\langle X\rangle$ denote the Lie subalgebra of $\mathcal{L} \otimes \mathbb{Q}$ generated by $X$. Let

$$
E_{n}=M_{\mathbb{Q}}^{\prime} \cap\left(\bigoplus_{i=1}^{n} \mathcal{L}_{i} \otimes \mathbb{Q}\right)
$$

and let $E_{n}^{\prime}=E_{n} \cap\left\langle E_{n-1}\right\rangle$. If we let $X_{n}:=$ a set of generators (as a $\mathbb{Q}$ vector space) for $E_{n} \bmod E_{n}^{\prime}$, then $X=\bigcup_{n} X_{n}$ is a free generating set of $M_{\mathbb{Q}}^{\prime}$.

We now show the afore-mentioned generators of $M_{\mathbb{Q}}^{\prime}$ to be free. Clearly, we can set $X_{1}:=\left\{d_{i}\right\}_{i=1}^{2 g_{2}}$. The only question is whether $\tilde{\gamma}$ is in the Lie algebra generated by $X_{1}$. Recall that $\tilde{\gamma}=\prod_{i=1}^{g_{1}}\left[c_{i}, c_{i+g_{1}}\right]$ and so in $\mathcal{L}_{2}$, we have $\tilde{\gamma}=\sum_{i=1}^{g_{1}}\left[c_{i}, c_{i+g_{1}}\right]$. As elements of $H$, the $c_{i}$ and $d_{i}$ freely generate $\mathcal{L} \otimes \mathbb{Q}$, so $\tilde{\gamma} \notin\left\langle X_{1}\right\rangle$. Thus, we can set $X_{2}=\{\tilde{\gamma}\}$, and so $\left\{d_{i}\right\}_{i=1}^{2 g_{2}} \cup\{\tilde{\gamma}\}$ freely generates $M_{\mathbb{Q}}^{\prime}$. But then clearly it freely generates $M^{\prime}$.

Now, we can define an inverse Lie homomorphism $M^{\prime} \rightarrow N$ by sending generators to generators, and so $N \rightarrow \mathcal{L}$ is injective. This proves Proposition 5.2.

By the proposition, we have $\Lambda_{n} \backslash \Lambda_{n+1} \hookrightarrow \Gamma_{n} \backslash \Gamma_{n+1}$, but this implies that in fact $\Lambda_{n}=\Lambda \cap \Gamma_{n}$. Thus, $M=M^{\prime}$.

Step 3 Recall that $C=$ image of $H_{1}\left(S_{1}\right)$ and $D=$ image of $H_{1}\left(S_{2}\right)$ in $H$; ie, $C=\left\langle\left\{c_{i}\right\}_{i=1}^{2 g_{1}}\right\rangle$ and $D=\left\langle\left\{d_{i}\right\}_{i=1}^{2\left(g_{2}\right)}\right\rangle$. Suppose $x \in D$. Then, by Steps 1 and 2 ,

$$
y:= \begin{cases}\tau_{f}(x) & k \text { even } \\ \tau_{f}^{2}(x) & k \text { odd }\end{cases}
$$

is an element of $M$. We can write $\tilde{\gamma}$ in $\mathcal{A}$ as $\sum_{i=1}^{2 g_{1}}\left(c_{i} \otimes c_{i+g_{1}}-c_{i+g_{1}} \otimes c_{i}\right)$. Thus, $M$ is contained in the subring generated by:

$$
\left\{\sum_{i=1}^{2 g_{1}}\left(c_{i} \otimes c_{i+g_{1}}-c_{i+g_{1}} \otimes c_{i}\right)\right\} \cup\left\{d_{i}\right\}_{i=1}^{2\left(g_{2}\right)}
$$

Consequently, we can write $y=\sum_{m} y_{m, 1} \otimes \cdots \otimes y_{m, n}$ where an even number of the elements of $\left\{y_{m, 1}, \ldots, y_{m, n}\right\}$ are in $C$ and the rest are in $D$. Since $\hat{\imath}\left(c_{i}, d_{j}\right)=0$ for all $i, j$, we have $\Phi_{n-1}\left(y_{m, 1} \otimes \cdots \otimes y_{m, n}\right) \neq 0$ only if $y_{m, n} \in D$. Thus, $\Psi_{k}(f)(D) \subseteq D$, and we are done with Claim 1.

Proof of Claim 2 Let $\alpha$ be the nonseparating curve which is fixed by $f \in \mathcal{I}_{k}$. Let $\widehat{S}$ be the surface obtained by cutting along $\alpha$, and $j: \widehat{S} \hookrightarrow S$ the canonical immersion. 
Similar to the proof of Claim 1, we will show that $\Psi_{k}(f)(C) \subseteq C$ where $C:=$ image of $H_{1}(\widehat{S}, \mathbb{Z})$ in $H$. Analagous to the above, we let

$$
M:=\bigoplus_{n} \hat{\Gamma} \cap \Gamma_{n} / \widehat{\Gamma} \cap \Gamma_{n+1}
$$

where $\widehat{\Gamma}=\pi_{1}(\widehat{S})$. We go through the same 3 steps as in the proof of Claim 1:

- Step 1 Show that $\tau_{f}(M) \subseteq M$.

- Step 2 Show that $M$ is a Lie subalgebra of $\mathcal{L}$ and find generators.

- Step 3 Show that $\Phi_{k}\left(M \cap \mathcal{L}_{k+1}\right) \subseteq C$.

Let us first set up some notation. Let $\alpha_{1}$ and $\alpha_{2}$ be the boundary curves of $\hat{S}$ such that $j_{*}\left(\alpha_{1}\right)=j_{*}\left(\alpha_{2}\right)=\alpha$. Choose based representatives $a, a_{1}$ and $a_{2}$ of $\alpha, \alpha_{1}$ and $\alpha_{2}$ respectively as in Figure 2; in particular, $j_{*}\left(a_{1}\right)=a$. Also, let $b$ be as depicted in Figure 2. Extend $\{a, b\}$ to a "standard" generating set $\{a, b\} \cup\left\{c_{i}\right\}_{i=1}^{2(g-1)}$; ie, the following hold:

(a) The set $\{a, b\} \cup\left\{c_{i}\right\}_{i=1}^{2(g-1)}$ gives a symplectic basis in homology.

(b) $\iota(a, b)=\hat{\imath}(a, b)=1$.

(c) All $c_{i}$ can be homotoped to lie entirely inside the interior of $\widehat{S}$.

Letting $a_{1}$ and $a_{2}$ be as in Figure 2, one can easily check that $j_{*}\left(a_{1} a_{2}^{-1}\right)=\left[a, b^{-1}\right]$.

Step 1 Choosing the same basepoint for $\widehat{S}$ and $S$, we have that $j_{*}: \pi_{1}(\widehat{S}) \rightarrow \pi_{1}(S)$ is injective and $\hat{\Gamma}=\pi_{1}(\hat{S})$ is invariant under $f_{*}$. Thus, we have

$$
M:=\bigoplus_{n} \hat{\Gamma} \cap \Gamma_{n} / \hat{\Gamma} \cap \Gamma_{n+1}
$$

is a $\tau_{f}$-invariant submodule of $\mathcal{L}$. It is also easy to see $M \cap \mathcal{L}_{1}=C$.

Step 2 Just as in the proof of Claim 1, we choose a filtration of $\pi_{1}(\widehat{S})$ which is a slight alteration of the lower central series:

$$
\begin{aligned}
& \widehat{\Gamma}_{1}=\pi_{1} \widehat{S} \\
& \widehat{\Gamma}_{2}=\left\langle\left[\widehat{\Gamma}_{1}, \widehat{\Gamma}_{1}\right], a_{1} a_{2}^{-1}\right\rangle \\
& \hat{\Gamma}_{n}=\left\langle\left[\widehat{\Gamma}_{n-k}, \widehat{\Gamma}_{k}\right]\right\rangle_{k=1}^{\left\lfloor\frac{m}{2}\right\rfloor} n \geq 3
\end{aligned}
$$

By Theorem 2.1, we get a corresponding graded $\mathbb{Z}$-Lie algebra which we denote by $\hat{M}$. Again, since $j_{*}\left(\widehat{\Gamma}_{n}\right) \subseteq \Gamma_{n}$, we get an induced Lie algebra homomorphism $\widehat{M} \rightarrow \mathcal{L}$. Note that $\widehat{M}$ is generated by $\left\{a_{1}\right\} \cup\left\{c_{i}\right\}_{i=1}^{2(g-1)} \in \widehat{M}_{1}$ and $a_{1} a_{2}^{-1} \in \widehat{M}_{2}$. Since $a_{1} a_{2}^{-1} \mapsto\left[a, b^{-1}\right]$, we have that $\left\{a,\left[a, b^{-1}\right]\right\} \cup\left\{c_{i}\right\}_{i=1}^{2(g-1)}$ generates $j_{*}(\hat{M})$. 


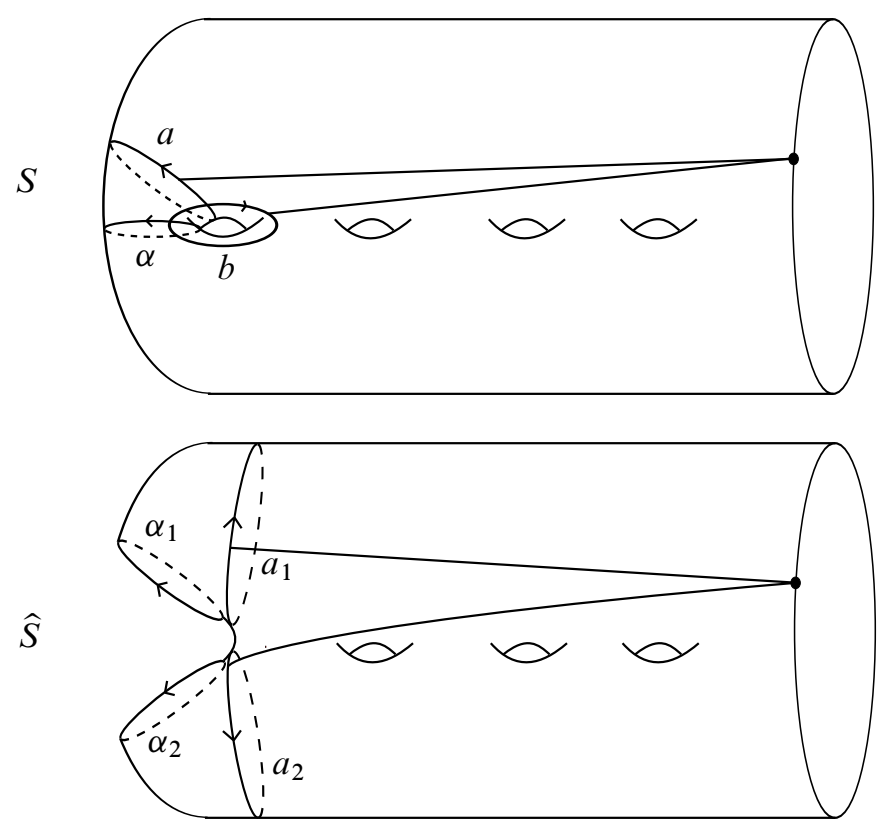

Figure 2

Proposition 5.3 The Lie algebra $\widehat{M}$ maps isomorphically onto $j_{*}(\widehat{M})$.

Proof of Proposition 5.3 Since the set $\{a, b\} \cup\left\{c_{i}\right\}_{i=1}^{2(g-1)}$ is a free generating set of $\mathcal{L}$, we have $\left[a, b^{-1}\right] \notin\left\langle a,\left\{c_{i}\right\}_{i=1}^{2(g-1)}\right\rangle$. Thus, by reasoning similar to that in the separating case, $\left\{a,\left[a, b^{-1}\right]\right\} \cup\left\{c_{i}\right\}_{i=1}^{2(g-1)}$ is a free generating set of $j_{*}(\hat{M})$. We obtain an inverse Lie algebra map $j_{*}(\widehat{M}) \rightarrow \widehat{M}$ induced by

$$
a \mapsto a_{1}, \quad\left[a, b^{-1}\right] \mapsto a_{1} a_{2}^{-1}, \quad c_{i} \mapsto c_{i}
$$

Since $\widehat{M}$ injects into $\mathcal{L}$, we have

$$
\widehat{\Gamma}_{m} \backslash \widehat{\Gamma}_{m+1} \hookrightarrow \Gamma_{m} \backslash \Gamma_{m+1}
$$

and so $\widehat{\Gamma}_{m}=\widehat{\Gamma} \cap \Gamma_{m}$. Thus, $j_{*}(\hat{M})=M$.

Step 3 Now, let $x \in C:=\left\langle a,\left\{c_{i}\right\}_{i=1}^{2(g-1)}\right\rangle \subseteq H$. Then

$$
y:= \begin{cases}\tau_{f}(x) & k \text { even } \\ \tau_{f}^{2}(x) & k \text { odd }\end{cases}
$$

Algebraic $8 \mathcal{G}$ Geometric Topology, Volume 7 (2007) 
is an element of $M$. As an element of $\mathcal{A}$, we may write $y=\sum_{m} y_{m, 1} \otimes \cdots \otimes y_{m, n}$ where each $y_{m, r}$ is a multiple of one of $a, b, c_{i}$. Since $[a, b]=a \otimes b-b \otimes a$ as an element of $\mathcal{A}$ and $y \in M$, there are at least as many $a$ terms as $b$ terms in $y_{m, 1}, \ldots, y_{m, n}$. Since $b$ pairs nontrivially only with $a$ in the set $\{a, b\} \cup\left\{c_{i}\right\}_{i=1}^{2(g-1)}$, we have $\Phi_{n-1}\left(y_{m, 1} \otimes \cdots \otimes y_{m, n}\right) \neq 0$ only if $y_{m, n} \neq$ a multiple of $b$, in which case $\Phi_{n-1}\left(\left(y_{m, 1} \otimes \cdots \otimes y_{m, n}\right) \in C\right.$. Thus, $\Psi_{k}(f)(C) \subseteq C$, and since $C$ has rank $2 g-1$, the characteristic polynomial of $\Psi_{k}(f)$ factors into a product of a degree 1 and degree $2 g-1$ polynomial.

\section{Theorem 1.2 versus the Thurston-Penner criteria}

In this section we will compare the criterion of Theorem 1.2 to the Thurston-Penner criteria. Since the Thurston-Penner criteria are topological and Theorem 1.2 is algebraic, one might expect that there is essentially no relation between the two. We will show this to be true in the following sense. There exist examples satisfying the Thurston or Penner criteria but not the hypothesis of Theorem 1.2 and examples satisfying both. As of the writing of this paper, it has not been proven that there are examples of pseudo-Anosovs which do not satisfy the Thurston-Penner criteria. However, we will give an example satisfying the hypothesis of Theorem 1.2 to which the Thurson-Penner criteria do not seem to apply directly.

Since we will be dealing with Dehn twists about separating curves, we first describe $\Psi_{2}\left(T_{\gamma}\right)$ where $\gamma$ is one of the $\gamma_{i}$ in Figure 3 and $T_{\gamma}$ is the Dehn twist about $\gamma$. First let us set up a symplectic basis. Let $\left\{\alpha_{i}, \beta_{i}\right\}$ be the curves as depicted in Figure 3 with $a_{i}=\left[\alpha_{i}\right]$ and $b_{i}=\left[\beta_{i}\right]$ their homology classes. Our ordered basis of $H$ throughout this section will be $\left\{a_{1}, b_{1}, a_{2}, b_{2}, \ldots, a_{g}, b_{g}\right\}$.

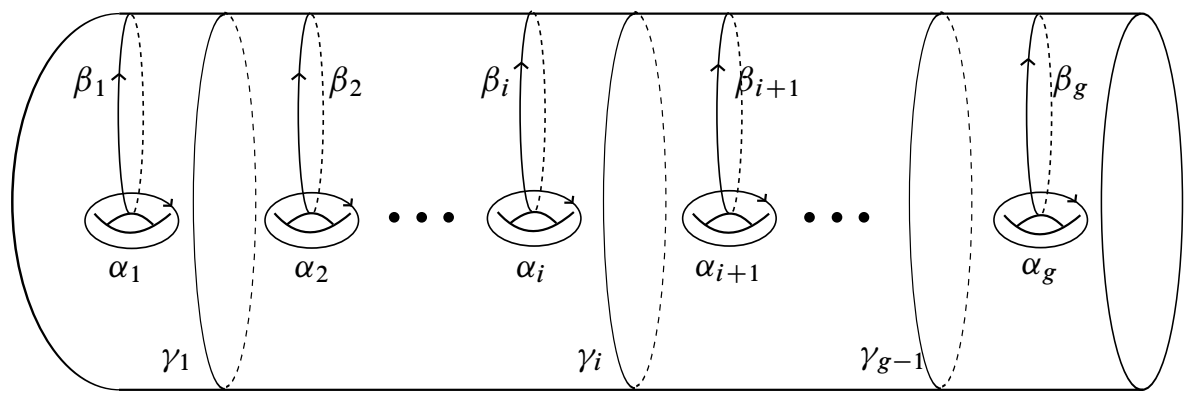

Figure 3

Algebraic 83 Geometric Topology, Volume 7 (2007) 
Lemma 6.1 With $\left\{a_{i}, b_{i}\right\}$ and $\left\{\gamma_{i}\right\}$ as above, the element $\Psi_{2}\left(T_{\gamma_{i}}\right) \in \operatorname{End}(H)$ is the map defined by:

$$
\begin{array}{r}
a_{j} \mapsto \begin{cases}(2 i+1) a_{j} & j \leq i \\
0 & j>i\end{cases} \\
b_{j} \mapsto \begin{cases}(2 i+1) b_{j} & j \leq i \\
0 & j>i\end{cases}
\end{array}
$$

Note that with the given indexing, $i$ is the genus of $\gamma_{i}$.

Proof We can lift $a_{i}, b_{i}, \gamma_{i}$ to $\tilde{a_{i}}, \tilde{b_{i}}, \tilde{\gamma_{i}} \in \pi_{1}(S)$ by connecting $\alpha_{i}, \beta_{i}$ and $\gamma_{i}$ to the basepoint via paths. Furthermore, we can do it in such a way that $\tilde{\gamma_{i}}=\prod_{j=1}^{i}\left[\tilde{a_{j}}, \tilde{b_{j}}\right]$ in $\pi_{1}(S)$ and

$$
\begin{aligned}
& T_{\gamma_{i}}\left(\tilde{a_{j}}\right)= \begin{cases}\tilde{\gamma_{i}}{\tilde{a_{j}}}^{\tilde{\gamma}_{i}}{ }^{-1} & j \leq i \\
\tilde{a_{j}} & j>i\end{cases} \\
& T_{\gamma_{i}}\left(\tilde{b_{j}}\right)= \begin{cases}\tilde{\gamma_{i}} \tilde{b}_{j}{\tilde{\gamma_{i}}}^{-1} & j \leq i \\
\widetilde{b_{j}} & j>i\end{cases}
\end{aligned}
$$

Thus, for $j \leq i$ and $f_{i}=T_{\gamma_{i}}$, we compute $f_{i}\left(\tilde{a_{j}}\right){\tilde{a_{j}}}^{-1}=\left[\tilde{\gamma_{i}}, \tilde{a_{j}}\right]$ and $\tau_{f_{i}}\left(a_{j}\right)=\left[\sum_{k=1}^{i}\left[a_{k}, b_{k}\right], a_{j}\right]=\sum_{k=1}^{i}\left(\left(a_{k} \otimes b_{k}-b_{k} \otimes a_{k}\right) \otimes a_{j}-a_{j} \otimes\left(a_{k} \otimes b_{k}-b_{k} \otimes a_{k}\right)\right)$

For $j>i$, we easily see that $\tau_{f_{i}}\left(a_{j}\right)=0$. Recall that $\Phi_{2}\left(c_{1} \otimes c_{2} \otimes c_{3}\right)=\hat{\imath}\left(c_{1}, c_{2}\right) c_{3}$. We then compute for $j \leq i$ that $\Psi_{2}\left(f_{i}\right)=\Phi_{2}\left(\tau_{f_{i}}\left(a_{j}\right)\right)=(2 i+1) a_{j}$. Clearly, $\Phi_{2}\left(\tau_{f_{i}}\left(a_{j}\right)\right)=0$ for $j>i$. The computation for $b_{j}$ is the same but with the roles of $a$ and $b$ switched.

Now let us consider $T_{\gamma}$ where $\gamma$ is an arbitrary separating curve not homotopic to the boundary. Recall that $\Psi_{k}$ is $\operatorname{Mod}(S)$-equivariant (This follows from the $\operatorname{Mod}(S)-$ equivariance of $\Phi_{k}$ and $\tau$ ). The $\operatorname{Mod}(S)$-action on $\operatorname{End}(H)$ is as follows. If $\varphi \in$ $\operatorname{Mod}(S)$ and $h \in \operatorname{End}(H)$, then

$$
\varphi \cdot h=[\varphi] h[\varphi]^{-1}
$$

where $[\varphi]$ denotes the projection of $\Phi$ to $\operatorname{Sp}(2 g, \mathbb{Z})$. Thus, for $f \in \mathcal{I}_{2}$ and $\varphi \in \operatorname{Mod}(S)$, we find that $\Psi_{k}\left(\varphi f \varphi^{-1}\right)=[\varphi] \Psi_{k}(f)[\varphi]^{-1}$. Recall that if for a fixed $g^{\prime}$, two separating curves $\eta_{1}$ and $\eta_{2}$ both cut $S$ into a $\Sigma_{g^{\prime}, 1}$ and a $\Sigma_{g-g^{\prime}, 2}$, then there is some $\varphi \in \operatorname{Mod}(S)$ such that $\varphi\left(\eta_{1}\right)=\eta_{2}$. Thus, $\Psi_{2}\left(T_{\gamma}\right)$ is of the form $\varphi \Psi_{2}\left(T_{\gamma_{i}}\right) \varphi^{-1}$ for some $i$ and 
some $\varphi \in \operatorname{Sp}(2 g, \mathbb{Z})$. Similarly, if $A$ is a multicurve of separating curves and $T_{A}$ the multicurve twist, then

$$
\Psi_{2}\left(T_{A}\right)=\varphi \Psi_{2}\left(\prod_{k=1}^{m} T_{\gamma_{i_{k}}}\right) \varphi^{-1}
$$

for some $\varphi \in \operatorname{Sp}(2 g, \mathbb{Z})$ and some subset $\left\{\gamma_{i_{k}}\right\}$ of $\left\{\gamma_{i}\right\}$.

For the reader's convenience, we recall a few definitions and state a corollary to both the Thurston and Penner criteria. A pants decomposition is a maximal set of pairwise nonisotopic simple closed curves which are pairwise disjoint and not null-homotopic. For an $S_{g, b}$, a pants decomposition consists of $3 g-3+2 b$ curves and contains all the boundary curves. Recall that a simple closed curve $\gamma$ is essential if it is neither homotopically trivial nor homotopic to a boundary component. We say that two curves $\eta$ and $v$ fill a surface $S$ if, for any essential simple closed curve $\gamma$, the curve $\gamma$ either intersects $\eta$ or $v$ nontrivially. We define the notion of filling for two multicurves similarly.

Corollary 6.2 (Thurston, Penner) If two multicurves $A$ and $B$ fill a surface, then the product of multicurve twists $T_{A} T_{B}^{-1}$ is pseudo-Anosov.

\subsection{Negative results for Theorem 1.2}

In this section we show that there is a pseudo-Anosov in $\mathcal{I}_{2}\left(S_{g, 1}\right)$ for each $g \geq 2$ which satisfies the Thurston-Penner criteria but not the hypothesis of Theorem 1.2. Let $T_{\gamma}$ denote the twist about a simple closed curve $\gamma$.

Theorem 6.3 For each $g \geq 2$, there exists two simple closed curves $\gamma_{g, 1}$ and $\gamma_{g, 2}$ filling $S=S_{g, 1}$ such that $f_{g}:=T_{\gamma_{g, 1}} T_{\gamma_{g, 2}}^{-1}$ does not satisfy the hypothesis of Theorem 1.2. However, by the Thurston-Penner criteria, we know $f_{g}$ is pseudo-Anosov.

Proof We break the proof into two cases. For $g=2$, we will explicitly compute $\Psi_{2}\left(f_{2}\right)$. For $g \geq 3$, the main idea is to show that there is an $f_{g}^{\prime}$ such that $f_{g}^{\prime}$ is reducible and $\Psi_{2}\left(f_{g}^{\prime}\right)=\Psi_{2}\left(f_{g}\right)$. Of course, then it is impossible for $\Psi_{2}\left(f_{g}\right)$ to satisfy the hypothesis of Theorem 1.2 since $\Psi_{2}\left(f_{g}^{\prime}\right)$ does not.

We also need a consequence of [8, Lemma 2 of Expose 13] to construct the $f_{g}$. For the reader's convenience, we state the consequence.

Lemma 6.4 Let $S$ be a surface. Let $\gamma$ be a simple closed curve on $S$ and $P=$ $\left\{\alpha_{1}, \ldots, \alpha_{m}\right\}$ a pants decomposition of $S$ such that $\iota\left(\gamma, \alpha_{i}\right) \neq 0$ for all $\alpha_{i}$ that are not boundary components. Then, the curves $\gamma$ and $T_{P}(\gamma)$ fill the surface. 
The case $g=2$ Let $\gamma_{2,1}$ and the $\eta_{i}$ be as in Figure 4. Since the $\eta_{i}$ are disjoint and $\left\{\eta_{i}\right\}$ is a 4 element set, $P=\left\{\eta_{i}\right\}$ is a pants decomposition. By Lemma 6.4, we know that $\gamma_{2,1}$ and $\gamma_{2,2}:=T_{P}(\gamma)$ fill $S$.

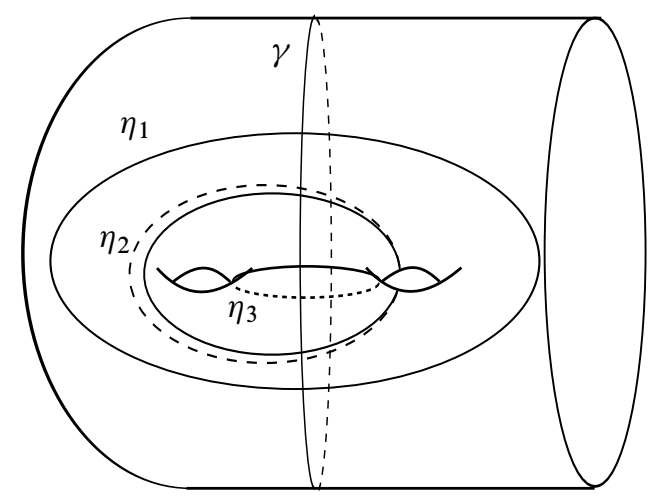

Figure 4

We now explicitly compute $\Psi_{2}\left(f_{2}\right)$ and see that its characteristic polynomial has degree 2 factors. Since $\Psi_{2}$ is a homomorphism and $\operatorname{Mod}(S)$-equivariant, we find that:

$$
\begin{aligned}
\Psi_{2}\left(T_{\gamma_{2,1}} T_{T_{P}\left(\gamma_{2,1}\right)}^{-1}\right)= & \Psi_{2}\left(T_{\gamma_{2,1}}\right)-\left[T_{P}\right] \circ \Psi_{2}\left(T_{\gamma_{2,1}}\right) \circ\left[T_{P}\right]^{-1} \\
= & \Psi_{2}\left(T_{\gamma_{2,1}}\right)- \\
& {\left[T_{\eta_{1}}\right]\left[T_{\eta_{2}}\right]\left[T_{\eta_{3}}\right]\left[T_{\eta_{4}}\right] \Psi_{2}\left(T_{\gamma_{2,1}}\right)\left[T_{\eta_{4}}\right]^{-1}\left[T_{\eta_{3}}\right]^{-1}\left[T_{\eta_{2}}\right]^{-1}\left[T_{\eta_{1}}\right]^{-1} } \\
= & \Psi_{2}\left(T_{\gamma_{2,1}}\right)-\left[T_{\eta_{1}}\right]\left[T_{\eta_{3}}\right] \Psi_{2}\left(T_{\gamma_{2,1}}\right)\left[T_{\eta_{3}}\right]^{-1}\left[T_{\eta_{1}}\right]^{-1}
\end{aligned}
$$

Note that since $\eta_{2}$ is separating, $\left[T_{\eta_{2}}\right]$ is trivial. For any simple closed curve $\beta$ and $c \in H$, one can show that

$$
\left[T_{\beta}\right](c)=c+\hat{\imath}([\beta], c)[\beta]
$$

where $[\beta]$ is the homology class of $\beta$. We see that $\left[\eta_{1}\right]=a_{1}+a_{2}$ and $\left[\eta_{3}\right]=b_{2}-b_{1}$ and so one computes:

$$
\Psi_{2}\left(T_{\gamma_{2,1}} T_{T_{P}\left(\gamma_{2,1}\right)}^{-1}\right)=3 *\left(\begin{array}{rrrr}
-1 & 0 & 1 & 1 \\
0 & -1 & 1 & -1 \\
-1 & -1 & 1 & 0 \\
-1 & 1 & 0 & 1
\end{array}\right)
$$

The characteristic polynomial is computed to be $\left(9+x^{2}\right)^{2}$.

The case $g \geq 3$ First, we find a pair of filling curves using Lemma 6.4. We prove the case of $g=5$ with the generalization to the general case of $g \geq 3$ being clear. Let $\gamma_{5,1}$ 
be the curve $\gamma_{1}$ in Figure 3 and $P$ the pants decomposition given by all the curves in Figure 6 and all the curves in Figure 5 except $v$. One sees that $\gamma_{5,1}$ intersects every curve of $P$ nontrivially. Thus, by Lemma $6.4, \gamma_{5,1}$ and $\gamma_{5,2}:=T_{P}\left(\gamma_{5,1}\right)$ fill $S_{5,1}$.

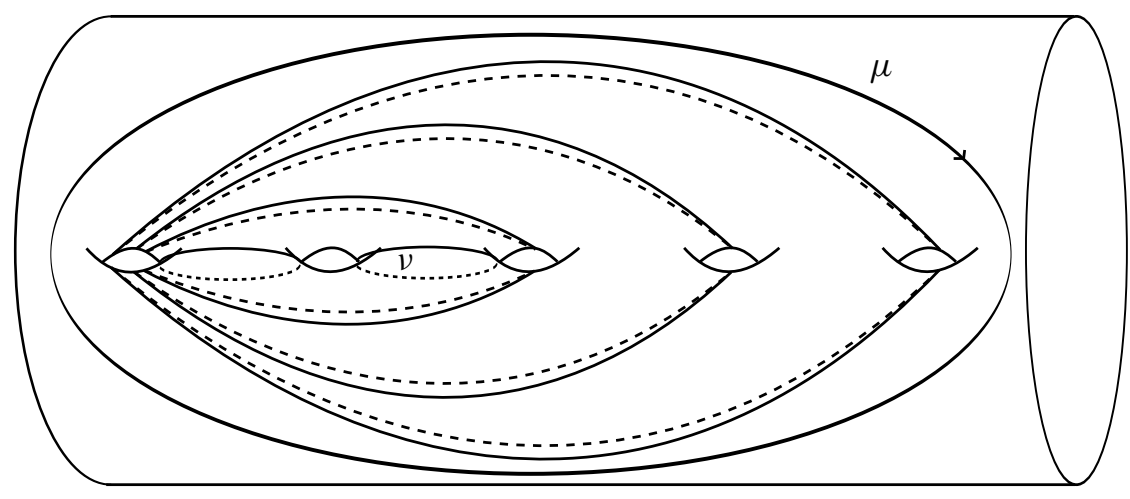

Figure 5

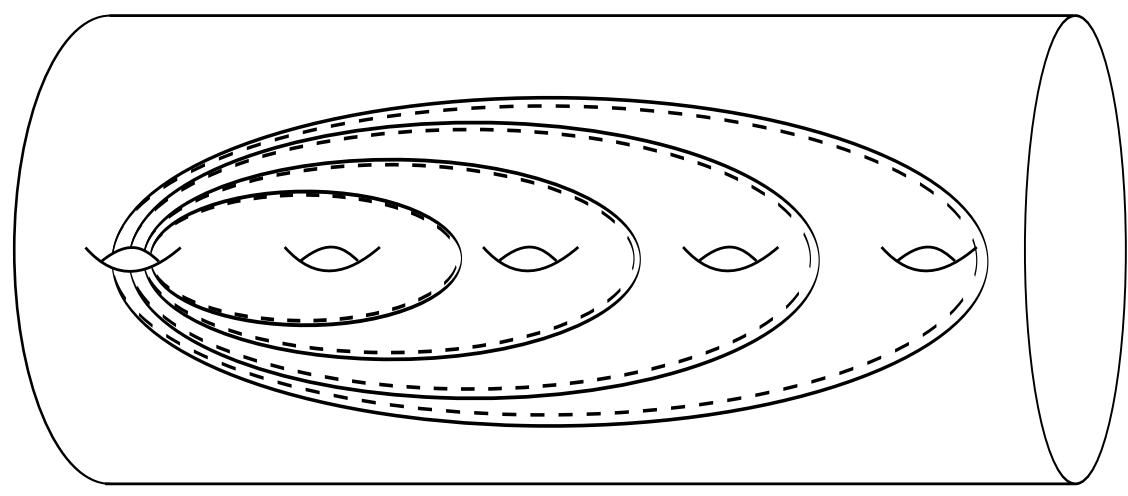

Figure 6

We next show that there is some $f_{5}^{\prime} \in \mathcal{I}_{2}$ such that $\Psi_{2}\left(f_{5}^{\prime}\right)=\Psi_{2}\left(f_{5}\right)$ and $f_{5}^{\prime}$ is reducible. Let

$$
P_{\text {nosep }}=\{\eta \in P \mid \eta \text { is nonseparating }\} .
$$

Since $\left[T_{\eta}\right]=I d$ for all $\eta$ that are separating, we see that:

$$
\begin{aligned}
\Psi_{2}\left(f_{5}\right) & =\Psi_{2}\left(T_{\gamma_{5,1}} T_{T_{P}\left(\gamma_{5,1}\right)}^{-1}\right)=\Psi_{2}\left(T_{\gamma_{5,1}}\right)-\left[T_{P}\right] \Psi_{2}\left(T_{\gamma_{5,1}}\right)\left[T_{P}^{-1}\right] \\
& =\Psi_{2}\left(T_{\gamma_{5,1}}\right)-\left[T_{P_{\text {nosep }}}\right] \Psi_{2}\left(T_{\gamma_{5,1}}\right)\left[T_{P_{\text {nosep }}}^{-1}\right]=\Psi_{2}\left(T_{\gamma_{5,1}} T_{P_{P_{\text {nosep }}}\left(\gamma_{5,1}\right)}^{-1}\right)
\end{aligned}
$$


We let $f_{5}^{\prime}=T_{\gamma_{5,1}} T_{T_{P_{\text {nosep }}}}^{-1}\left(\gamma_{5,1}\right)$. Notice that the curve $v$ in Figure 5 intersects neither $\gamma_{5,1}$ nor $T_{P_{\text {nosep }}}\left(\gamma_{5,1}\right)$, and so $f_{5}^{\prime}(v)=v$. Thus, $f_{5}^{\prime}$ is reducible.

There is one more subtle point we must show to prove that in fact $f_{5}$ cannot be shown to be pseudo-Anosov using Theorem 1.2. We need to establish that $f_{5} \in \mathcal{I}_{2} \backslash \mathcal{I}_{3}$ since, if $f_{5} \in \mathcal{I}_{3}$, we can then apply $\Psi_{3}$ and perhaps $\Psi_{3}\left(f_{5}\right)$ has irreducible characteristic polynomial. Since $\Psi_{2}\left(\mathcal{I}_{3}\right)=0$, it is sufficient to prove $\Psi_{2}\left(f_{5}\right) \neq 0$. Suppose $\Psi_{2}\left(f_{5}\right)=$ 0 . Then we have that:

$$
\Psi_{2}\left(T_{\gamma_{5,1}}\right)-\left[T_{P_{\text {nosep }}}\right] \Psi_{2}\left(T_{\gamma_{5,1}}\right)\left[T_{P_{\text {nosep }}}^{-1}\right]=0
$$

In other words, the endomorphisms $\left[T_{P_{\text {nosep }}}\right]$ and $\Psi_{2}\left(T_{\gamma_{5,1}}\right)$ commute. Letting $e_{i, j}$ be the elementary matrix with a 1 in the $(i, j)$ th entry and 0 's everywhere else, Lemma 6.1 implies $\Psi_{2}\left(T_{\gamma_{5,1}}\right)=e_{1,1}+e_{2,2}$ as a matrix in our chosen basis. Commuting with $e_{1,1}+e_{2,2}$ implies that the $(1,10)$ th entry of $\left[T_{P_{\text {nosep }}}\right]$ is 0 ; ie, the $a_{1}$ coefficient of $\left[T_{P_{\text {nosep }}}\right]\left(b_{5}\right)$ is 0 . Since the only curve in Figure 5 that intersects $\beta_{5}$ nontrivially is $\mu$, we have that

$$
\left[T_{P_{\text {nosep }}}\right]\left(b_{5}\right)=\left[T_{\mu}\right]\left(b_{5}\right)=b_{5}+\widehat{\imath}\left(b_{5},[\mu]\right)[\mu]=b_{5}-\sum_{i=1}^{5} a_{i}
$$

which gives a contradiction. (For a proof of the second equality, see Farb and Margalit [7, Section 6.1.3].)

\subsection{Positive results for Theorem 1.2}

In this section, we will exhibit two examples of mapping classes which satisfy the hypothesis of Theorem 1.2. Both examples were found through a computer search. We begin with an example satisfying both Theorem 1.2 and the Thurston-Penner criteria.

We first make some preliminary remarks. If $A$ and $B$ are multicurves and $T_{A} T_{B}^{-1}$ is pseudo-Anosov, then it is clear that $A \cup B$ fills $S$. Thus, if $T_{A} T_{B}^{-1}$ satisfies the hypothesis of Theorem 1.2, it immediately follows that the Thurston-Penner criteria imply that $T_{A} T_{B}^{-1}$ is pseudo-Anosov.

Now let us describe our example explicitly. Let $S=S_{5,1}$. We let $A=\left\{\gamma_{1}, \gamma_{2}, \gamma_{3}\right\}$ and $B^{\prime}=\left\{\gamma_{1}, \gamma_{2}\right\}$ where the $\gamma_{i}$ are the separating curves given in Figure 3. Let $h \in \operatorname{Mod}(S)$ 
be any mapping class such that its projection to $\operatorname{Sp}(2 g, \mathbb{Z})$ is given by:

$$
[h]=\left(\begin{array}{rrrrrrrrrr}
2 & 0 & 1 & 1 & 1 & 1 & 2 & 0 & 1 & 0 \\
1 & 2 & -2 & 0 & 0 & -1 & 1 & -1 & 2 & -2 \\
3 & 3 & 2 & -1 & 2 & 0 & 0 & 1 & 2 & -3 \\
1 & -1 & 0 & 2 & 1 & 0 & 2 & 0 & 1 & 1 \\
4 & 3 & 2 & -1 & 2 & 1 & 1 & 0 & 2 & -2 \\
0 & -1 & 2 & 0 & 0 & 1 & 0 & 1 & -1 & 1 \\
0 & -1 & 0 & 1 & 0 & 0 & 1 & 0 & 0 & 1 \\
6 & 0 & 7 & 2 & 5 & 2 & 3 & 4 & 2 & 0 \\
1 & -1 & 2 & 0 & 0 & 1 & 0 & 1 & 0 & 1 \\
1 & 0 & 1 & 0 & 0 & 0 & 0 & 0 & 0 & 1
\end{array}\right)
$$

Let $B=h\left(B^{\prime}\right)$. If we let $e_{i, j}$ be the elementary matrix with a 1 in the $(i, j)$ th entry and 0 's everywhere else, then using Lemma 6.1, we find

$$
T_{A}=15\left(e_{1,1}+e_{2,2}\right)+12\left(e_{3,3}+e_{4,4}\right)+7\left(e_{5,5}+e_{6,6}\right)
$$

and

$$
T_{B^{\prime}}=8\left(e_{1,1}+e_{2,2}\right)+5\left(e_{3,3}+e_{4,4}\right) .
$$

Putting this together, we compute (via Mathematica):

$$
\begin{aligned}
\Psi_{2}\left(T_{A} T_{B}^{-1}\right)= & \Psi\left(T_{A}\right)-[h] \Psi\left(T_{B^{\prime}}\right)[h]^{-1} \\
& =\left(\begin{array}{rrrrrrrrrr}
42 & 0 & -6 & -33 & -26 & -33 & -25 & 11 & -5 & 26 \\
0 & 42 & -44 & 14 & -8 & 30 & -116 & 18 & -16 & 24 \\
14 & 33 & -28 & 0 & -14 & 24 & -89 & 14 & -19 & 38 \\
44 & -6 & 0 & -28 & -28 & -36 & -22 & 8 & -2 & 20 \\
30 & 33 & -36 & -24 & -22 & 0 & -89 & 22 & -19 & 46 \\
8 & -26 & 28 & -14 & 0 & -22 & 68 & -10 & 8 & -8 \\
18 & -11 & 8 & -14 & -10 & -22 & 13 & 0 & 3 & 2 \\
116 & -25 & 22 & -89 & -68 & -89 & 0 & 13 & -10 & 68 \\
24 & -26 & 20 & -38 & -8 & -46 & 68 & -2 & 8 & 0 \\
16 & -5 & 2 & -19 & -8 & -19 & 10 & 3 & 0 & 8
\end{array}\right)
\end{aligned}
$$

We compute (via Mathematica) the characteristic polynomial to be

$$
\left(x^{5}-21 x^{4}+107 x^{3}+3837 x^{2}-13500 x+151200\right)^{2}
$$

and furthermore find that modulo 17 the polynomial

$$
x^{5}-21 x^{4}+107 x^{3}+3837 x^{2}-13500 x+151200
$$

Algebraic 83 Geometric Topology, Volume 7 (2007) 
is irreducible, and hence irreducible over $\mathbb{Z}$. Thus, by Theorem 1.2, $T_{A} T_{B}^{-1}$ is pseudoAnosov and we are done.

We now exhibit a mapping class $f \in \mathcal{I}_{1}\left(S_{4,1}\right)$ for which there is no obvious way to apply the Thurston-Penner criteria. First, let us recall some facts about the Johnson homomorphism on $\mathcal{I}_{1}$. There is the following sequence of canonical embeddings and isomorphisms:

$$
\Lambda^{3} H \hookrightarrow \Lambda^{2} H \otimes H \cong\left(\Gamma_{2} / \Gamma_{3}\right) \otimes H \cong \operatorname{Hom}\left(H, \Gamma_{2} / \Gamma_{3}\right)
$$

[10, Theorem 1] implies:

$$
\tau\left(\mathcal{I}_{1} / \mathcal{I}_{2}\right)=\operatorname{image}\left(\Lambda^{3} H\right) \subseteq \operatorname{Hom}\left(H, \Gamma_{2} / \Gamma_{3}\right)
$$

We define a bounding pair to be a pair of nonisotopic disjoint curves whose union separates the surface. The bounding pair map associated to an ordered bounding pair $(\eta, \gamma)$ is the product of Dehn twists $T_{\eta} T_{\gamma}^{-1}$. Let $h=T_{\beta_{i}} T_{\beta_{i}^{\prime}}^{-1}$ be the bounding pair map for $\beta_{i}$ and $\beta_{i}^{\prime}$ as given in Figure 7.

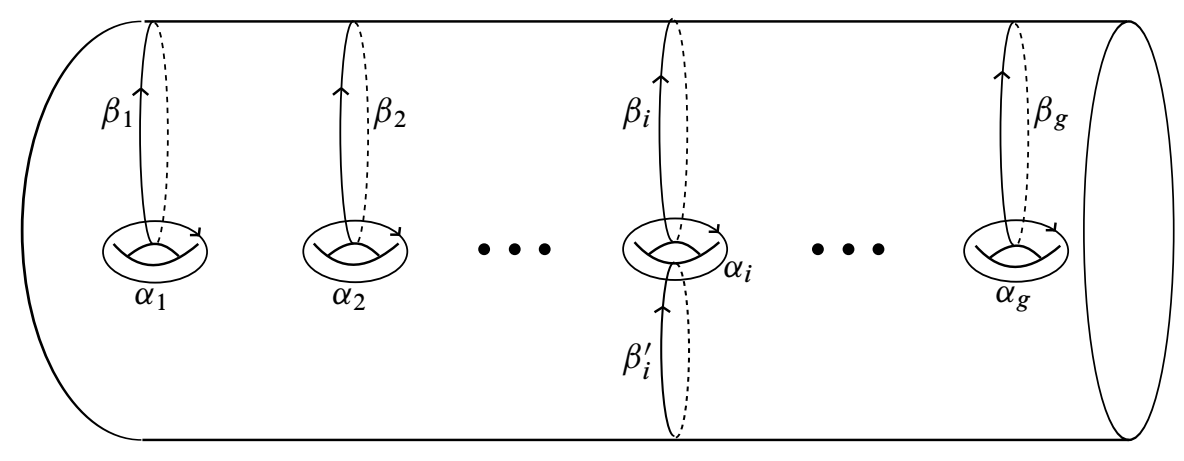

Figure 7

In $[10$, Lemma 4B], Johnson computes that

$$
\tau_{h}=\left(\sum_{j=1}^{i-1} a_{j} \wedge b_{j}\right) \wedge b_{i}
$$

Now, let us describe the example. Let

$$
\begin{aligned}
y=\left(a_{4}+b_{2}+b_{3}\right) \wedge a_{1} \wedge b_{1}+\left(a_{3}+b_{4}\right) \wedge a_{2} \wedge b_{2} \\
+\left(a_{1}+a_{2}+b_{1}\right) \wedge a_{3} \wedge b_{3}+\left(a_{1}+a_{2}\right) \wedge a_{4} \wedge b_{4} \in \Lambda^{2} H .
\end{aligned}
$$

Algebraic $8 \mathcal{G}$ Geometric Topology, Volume 7 (2007) 
From the previous paragraph, we know there exists $f \in \mathcal{I}$ such that $\tau_{f}=y$ which we construct now. Consider the bounding pairs illustrated in Figure 8 . Let $f$ be the product of bounding pair maps about these bounding pairs. Since $\tau$ is a homomorphism to an abelian group, $\tau_{f}$ is the same regardless of how the bounding pair maps are composed. Using (4), one computes that $\tau_{f}=y$.

$a$
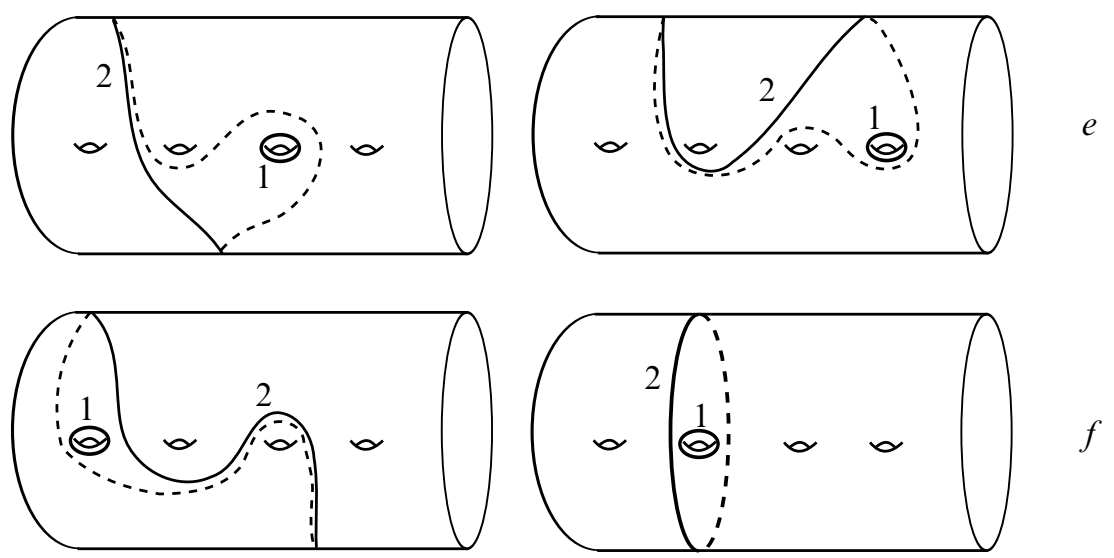

$c$
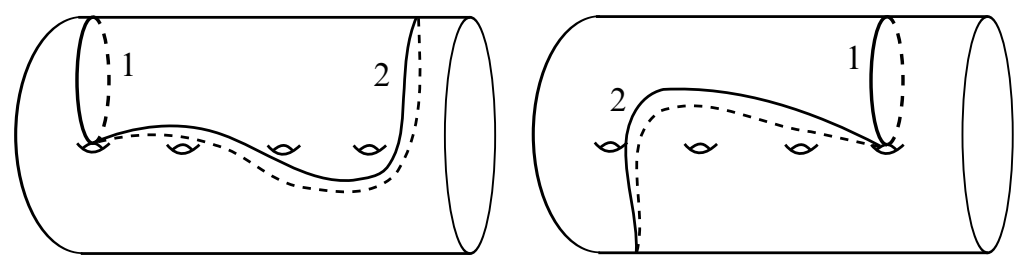

$g$
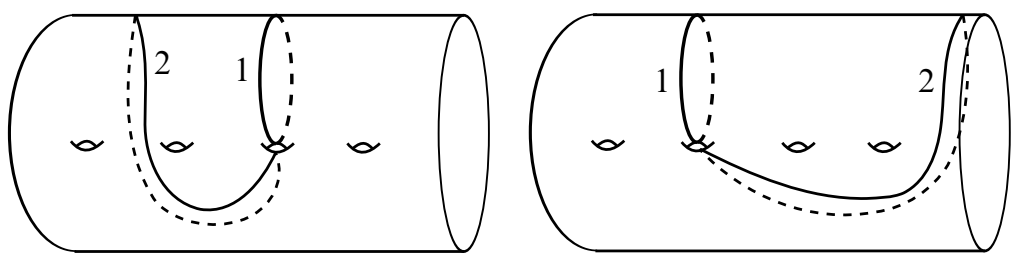

$h$

Figure 8: The product of the bounding pair maps indicated in a-h yields $y$

Via computation (with Mathematica), we find that with respect to the symplectic basis $\left\{a_{1}, b_{1}, \ldots, a_{4}, b_{4}\right\}$ : 


$$
\Psi_{1}(f)=\left(\begin{array}{rrrrrrrr}
-6 & -2 & 2 & 0 & 2 & 2 & -2 & 0 \\
4 & 2 & 2 & -2 & -2 & 2 & 2 & -2 \\
4 & -2 & 2 & 0 & -2 & 2 & 2 & 0 \\
-2 & 4 & -2 & 0 & 0 & 2 & 0 & 2 \\
-4 & -4 & 2 & 4 & -2 & 4 & 0 & -2 \\
-4 & -4 & 0 & 6 & 2 & 2 & -2 & 0 \\
-2 & 4 & -2 & 2 & 2 & 2 & 2 & 2 \\
4 & -2 & -2 & -4 & -4 & 2 & 4 & 0
\end{array}\right)
$$

The characteristic polynomial of $\Psi_{1}(f) / 2$ is

$$
\chi\left(\Psi_{1}(f) / 2\right)=x^{8}-8 x^{6}+26 x^{5}-18 x^{4}-76 x^{3}+241 x^{2}-558 x+553 .
$$

This polynomial is found to be irreducible mod 11 via Mathematica and is hence irreducible. By Theorem 1.2, $f$ is pseudo-Anosov. Note that curves $c_{2}, d_{2}$, and $g_{2}$ in Figure 8 all pairwise intersect, and so the criteria of Thurston and Penner do not seem to apply directly to $f$.

\section{References}

[1] H Bass, A Lubotzky, Linear-central filtrations on groups, from: "The mathematical legacy of Wilhelm Magnus: groups, geometry and special functions (Brooklyn, NY, 1992)", Contemp. Math. 169, Amer. Math. Soc. (1994) 45-98 MR1292897

[2] M Bestvina, M Handel, Train-tracks for surface homeomorphisms, Topology 34 (1995) 109-140 MR1308491

[3] P Brinkmann, XTrain Available at http://www.sci.ccny.cuny.edu/ $\sim$ brinkman/

[4] N Broaddus, B Farb, A Putman, Irreducible Sp-representations and subgroup distortion in the mapping class group, preprint

[5] A J Casson, S A Bleiler, Automorphisms of surfaces after Nielsen and Thurston, London Math. Soc. Student Texts 9, Cambridge University Press (1988) MR964685

[6] B Farb, C Leininger, D Margalit, The lower central series and pseudo-Anosov dilatations arXiv:math.GT/0603675

[7] B Farb, D Margalit, A primer on mapping class groups, in preparation

[8] A Fathi, F Laudenbach, V Poenaru, Travaux de Thurston sur les surfaces, Astérisque 66, Séminaire Orsay, with an English summary, Société Mathématique de France, Paris (1979) MR568308

[9] N V Ivanov, Subgroups of Teichmüller modular groups, Translations of Mathematical Monographs 115, Amer. Math. Soc. (1992) MR1195787 Translated from the Russian by E JF Primrose and revised by the author 
[10] D Johnson, An abelian quotient of the mapping class group $\mathcal{I}_{g}$, Math. Ann. 249 (1980) 225-242 MR579103

[11] W Magnus, A Karrass, D Solitar, Combinatorial group theory: Presentations of groups in terms of generators and relations, Interscience Publishers (John Wiley \& Sons) (1966) MR0207802

[12] S Morita, On the structure of the Torelli group and the Casson invariant, Topology 30 (1991) 603-621 MR1133875

[13] S Morita, Abelian quotients of subgroups of the mapping class group of surfaces, Duke Math. J. 70 (1993) 699-726 MR1224104

[14] S Morita, The extension of Johnson's homomorphism from the Torelli group to the mapping class group, Invent. Math. 111 (1993) 197-224 MR1193604

[15] S Morita, Structure of the mapping class groups of surfaces: a survey and a prospect, from: "Proceedings of the Kirbyfest (Berkeley, CA, 1998)", (J Hass, M Scharlemann, editors), Geom. Topol. Monogr. 2 (1999) 349-406 MR1734418

[16] R C Penner, A construction of pseudo-Anosov homeomorphisms, Trans. Amer. Math. Soc. 310 (1988) 179-197 MR930079

[17] C Reutenauer, Free Lie algebras, London Math. Soc. Monographs, New Series 7, Oxford University Press (1993) MR1231799

[18] W P Thurston, On the geometry and dynamics of diffeomorphisms of surfaces, Bull. Amer. Math. Soc. (N.S.) 19 (1988) 417-431 MR956596

Department of Mathematics, University of Chicago

5734 S University Ave, Chicago IL 60637, USA

justinm@math .uchicago . edu

www.math.uchicago.edu/ justinm/

Received: 7 March 2007 Revised: 17 July 2007 